REPRESENTATION THEORY

An Electronic Journal of the American Mathematical Society

Volume 12, Pages 294-326 (August 27, 2008)

S 1088-4165(08)00332-4

\title{
THE CENTER OF QUANTUM SYMMETRIC PAIR COIDEAL SUBALGEBRAS
}

\author{
STEFAN KOLB AND GAIL LETZTER
}

\begin{abstract}
The theory of quantum symmetric pairs as developed by the second author is based on coideal subalgebras of the quantized universal enveloping algebra for a semisimple Lie algebra. This paper investigates the center of these coideal subalgebras, proving that the center is a polynomial ring. A basis of the center is given in terms of a submonoid of the dominant integral weights.
\end{abstract}

\section{INTRODUCTION}

After the introduction of quantum groups in the mid 1980s it was natural to look for quantum analogs of compact symmetric spaces. The program was motivated by a desire to interpret I. G. Macdonald's large families of orthogonal polynomials Mac00 as zonal spherical functions on such quantum analogs. It became evident that quantum symmetric spaces were best described in terms of one-sided or twosided coideals inside the quantized universal enveloping algebra. We refer to this characterization as the theory of quantum symmetric pairs. By now, there exists a well established theory of quantum symmetric pairs and many of the initial questions have been answered.

There are two major approaches to the construction of quantum symmetric pairs. In a series of papers M. Noumi, T. Sugitani, and M.S. Dijkhuizen (Nou96], NS95, Dij96, DN98, DS99, and references therein) constructed them for all classical compact symmetric spaces. The constructions were done case-by-case and depended on solutions of the so-called reflection equation, which were given explicitly. Taking a unified approach, the second author classified and analyzed quantum symmetric pairs for all compact symmetric spaces (see [Let02, Let08] and references therein). The fundamental objects, quantum symmetric pair coideal subalgebras, were described explicitly in terms of generators and relations Let03. The reflection equation does not appear in this development.

By construction, quantum symmetric pair coideal subalgebras are quantum analogs of universal enveloping algebras of Lie subalgebras fixed by an involution inside a semisimple Lie algebra. These fixed Lie subalgebras are reductive. One expects a representation theory for their quantum analogs. At present, only special examples have been studied. For the symmetric pair of type AI, the representations were investigated in detail in [K05]. For the symmetric pair of type AIII,

Received by the editors February 27, 2006 and, in revised form, June 18, 2008.

2000 Mathematics Subject Classification. Primary 17B37.

The first author was supported by the German Research Foundation (DFG).

The second was supported by grants from the National Security Agency. 
certain representations were explicitly constructed in OS05. These representations were used to construct quantum analogs of sections of homogeneous vector bundles over Grassmann manifolds. This, in turn, led to new interpretations of Macdonald-Koornwinder polynomials.

The present paper can be viewed as the first step in developing a unified representation theory of quantum symmetric pair coideal subalgebras. We determine the center of such algebras. As in the classical case, it is shown that the center is a polynomial ring. More precisely, let $\theta$ be an involutive automorphism of a finite-dimensional complex semisimple Lie algebra $\mathfrak{g}$ and let $\mathfrak{g}^{\theta}$ denote the fixed Lie subalgebra. Let $\check{B}$ be a corresponding quantum symmetric pair coideal subalgebra inside the simply connected quantized universal enveloping algebra $\check{U}$ of $\mathfrak{g}$.

Theorem. The center $Z(\check{B})$ is a polynomial ring in rank $\left(\mathfrak{g}^{\theta}\right)$ variables.

Moreover, we show that $Z(\check{B})$ has a natural basis indexed by a subset $P_{Z(\check{B})}$ of the set of dominant integral weights for $\mathfrak{g}$. Just as for the ordinary quantized enveloping algebra, we expect central characters to be useful in the analysis of completely reducible $\check{B}$-modules and the classification of simple $\check{B}$-modules.

Part of our initial motivation in studying the center $Z(\breve{B})$ was to gain a better understanding of the relation between the two main constructions of quantum symmetric pairs. As explained in [Let99b, Section 6], Noumi-Sugitani-Dijkhuizen type coideals are contained in quantum symmetric pair coideal subalgebras. For the opposite direction, let $\operatorname{ad}_{r}$ denote the right adjoint action of $\breve{U}$ on itself and let $\tau(2 \mu) \in \check{U}$ be the group-like element corresponding to the dominant weight $2 \mu$. It was pointed out in [Kol] that any element in $Z(\check{B}) \cap\left(\operatorname{ad}_{r} \check{U}\right) \tau(2 \mu)$ leads to a solution of the reflection equation and hence associates a coideal subalgebra of Noumi-Sugitani-Dijkhuizen type to the quantum symmetric pair coideal subalgebra $\check{B}$. Thus suitable central elements in $\check{B}$ for exceptional $\mathfrak{g}$ allow Noumi-SugitaniDijkhuizen type constructions for compact symmetric spaces of exceptional type. The present paper guarantees the existence of such central elements.

There are several techniques for analyzing the center of quantized universal enveloping algebras. M. Rosso Ros90 and, similarly, C. De Concini and V.G. Kac CK90 used an analog of the Harish-Chandra homomorphism to describe the center. For quantum symmetric pair coideal subalgebras, however, it is not yet clear how to define an analog of a Cartan subalgebra. Thus a Harish-Chandra homomorphism is not available. In the present paper, we follow a different approach. A. Joseph and the second author JL94 determined all finite-dimensional submodules of $\check{U}$ under the adjoint action. The resulting locally finite part $F_{r}(\check{U})$ contains the center of $\check{U}$ in an immediately identifiable way. It was observed in Let99a that $Z(\check{B})$ is a subalgebra of $F_{r}(\check{U})$. Hence any central element in $\check{B}$ is contained in a sum of spherical submodules of $F_{r}(\check{U})$. To determine $Z(\check{B})$ we exploit the structure of the direct summand $\left(\operatorname{ad}_{r} \check{U}\right) \tau(2 \mu)$, for $\mu$ a dominant integral weight, of the locally finite part. Moreover, using the structure of $\check{B}$ and two filtrations invariant under the adjoint action, we show that $\left(\operatorname{ad}_{r} \check{U}\right) \tau(2 \mu)$ contains an element of $Z(\check{B})$ up to terms of lower filter degree if and only if $\mu \in P_{Z(\check{B})}$.

The situation considered here is reminiscent of an approach taken in FM98, [FMJ01] to determine the semicenter of the quantized enveloping algebra of a parabolic subalgebra of $\mathfrak{g}$. The semicenter is also contained in the locally finite part of $\check{U}$. It is noteworthy that the condition for $\left(\operatorname{ad}_{r} \check{U}\right) \tau(2 \mu)$ to contain an element in 
the semicenter [FM98, Théorème 3] resembles the condition in the definition (34) of $P_{Z(\check{B})}$.

This paper is organized in nine sections. After fixing notation, we briefly recall the construction and some properties of the quantum symmetric pair $\check{U}, \check{B}$. In Section 2, the filtration invariant under the adjoint action from JL94 is compared to a similar filtration based on the restricted root system of the symmetric pair. It is shown that the locally finite parts of the associated graded algebras coincide as $\check{U}$-modules. This will allow us to work with the second filtration when necessary.

In Section 3, we obtain preliminary properties of direct summands $\left(\operatorname{ad}_{r} \check{U}\right) \tau(2 \mu)$ of the locally finite part that contain highest weight components of central elements in $\check{B}$. A refinement of these properties leads to the definition of $P_{Z(\check{B})}$ in Section 7 .

There are two tensor product decompositions of $\check{U}$, the triangular and the quantum Iwasawa decomposition. Using the first, one can read off the minimal filter degree with respect to the filtrations introduced in Section 2 On the other hand, using the second, one easily checks whether an element belongs to $\check{B}$. In Sections 4 and [5] it is shown that weight estimates and filter degrees can also be read off from elements decomposed with respect to the quantum Iwasawa decomposition.

The second decomposition is also at the heart of producing a projection map from $\check{U}$ onto $\check{B}$ as defined in Section 6. Applying results from the previous sections, we show that this projection map preserves weight estimates and filter degrees. The projection map is then used to approximate general elements in $\check{U}$ by elements in $\check{B}$ with similar properties.

In Section 7 it is shown that for every $\mu \in P_{Z(\check{B})}$ there exists a unique highest weight vector of spherical weight in $\left(\operatorname{ad}_{r} \check{U}\right) \tau(2 \mu)$ that also occurs as a highest weight component of an element in $\check{B}$. This highest weight vector is the highest weight component of a central element $d_{\mu} \in Z(\check{B})$ constructed in Section 8 . We prove that $\left\{d_{\mu} \mid \mu \in P_{Z(\check{B})}\right\}$ is a basis of $Z(\check{B})$.

Finally, in Section 9 , the set $P_{Z(\breve{B})}$ is determined explicitly for every symmetric pair. It turns out that $P_{Z(\check{B})}$ is a free monoid of $\operatorname{rank} \operatorname{rank}\left(\mathfrak{g}^{\theta}\right)$. This result is used to show that $Z(\check{B})$ is a polynomial $\operatorname{ring}$ in $\operatorname{rank}\left(\mathfrak{g}^{\theta}\right)$ variables.

For the convenience of the reader we have listed all commonly used notation, in order of appearance, in an appendix.

\section{Preliminaries}

Let $\mathbb{C}$ denote the complex numbers, $\mathbb{Z}$ denote the integers, and $\mathbb{N}$ denote the nonnegative integers.

1.1. Complex semisimple Lie algebras. Let $\mathfrak{g}$ be a finite-dimensional complex semisimple Lie algebra of rank $n$ and $\mathfrak{h}$ a fixed Cartan subalgebra of $\mathfrak{g}$. Let $\Delta$ denote the root system associated with $(\mathfrak{g}, \mathfrak{h})$. Choose an ordered basis $\pi=\left\{\alpha_{1}, \ldots, \alpha_{n}\right\}$ of simple roots for $\Delta$. Let $W$ denote the Weyl group associated to the root system $\Delta$ and let $w_{0}$ denote the longest element in $W$ with respect to $\pi$. There is a unique $W$-invariant symmetric bilinear form $(\cdot, \cdot)$ on $\mathfrak{h}^{*}$ such that $(\alpha, \alpha)=2$ for all short roots $\alpha \in \Delta$. This form satisfies $(\alpha, \alpha) / 2 \in\{1,2,3\}$ for all $\alpha \in \Delta$. We write $Q(\pi)$ for the root lattice and $P(\pi)$ for the weight lattice associated to the root system $\Delta$. Set $Q^{+}(\pi)=\mathbb{N} \pi$ and let $P^{+}(\pi)$ be the set of dominant integral weights with respect to $\pi$. We will denote the fundamental weights in $P^{+}(\pi)$ by $\omega_{1} \ldots, \omega_{n}$. Let 
$\leq$ denote the standard partial ordering on $\mathfrak{h}^{*}$. In particular, $\mu \leq \gamma$ if and only if $\gamma-\mu \in Q^{+}(\pi)$.

1.2. The quantized enveloping algebra. Let $\mathcal{C}=\mathbb{C}(q)$ denote the field of rational functions in an indeterminate $q$. We consider here the $q$-deformed universal enveloping algebra $U_{q}(\mathfrak{g})$ as the $\mathcal{C}$-algebra generated by elements $t_{i}, t_{i}^{-1}, x_{i}, y_{i}$, where $i=1, \ldots, n$, subject to the relations as given in [Jos95, Section 3.2.9]. In particular, one has:

(i) The $t_{1}, \ldots, t_{n}$ generate a free abelian group of rank $n$.

(ii) $t_{i} x_{j}=q^{\left(\alpha_{i}, \alpha_{j}\right)} x_{j} t_{i}$ and $t_{i} y_{j}=q^{-\left(\alpha_{i}, \alpha_{j}\right)} y_{j} t_{i}$ for all $1 \leq i \leq n$.

(iii) $x_{i} y_{j}-y_{j} x_{i}=\delta_{i j}\left(t_{i}-t_{i}^{-1}\right) /\left(q^{\left(\alpha_{i}, \alpha_{i}\right) / 2}-q^{-\left(\alpha_{i}, \alpha_{i}\right) / 2}\right)$ for all $1 \leq i, j \leq n$.

(iv) The $x_{1}, \ldots, x_{n}$ and the $y_{1}, \ldots, y_{n}$ satisfy the quantum Serre relations (see for example [Let02, (1.7)]).

We often write $U=U_{q}(\mathfrak{g})$. Note that from Section 5.1 on we sometimes consider extensions of $U_{q}(\mathfrak{g})$ which are defined only over $\mathbb{C}\left(q^{1 / 2}\right)$.

The algebra $U$ also has a Hopf algebra structure with coproduct $\Delta$, antipode $\sigma$, and counit $\epsilon$ satisfying

$$
\begin{aligned}
& \Delta x_{i}=x_{i} \otimes 1+t_{i} \otimes x_{i}, \quad \sigma\left(x_{i}\right)=-t_{i}^{-1} x_{i}, \quad \epsilon\left(x_{i}\right)=0, \\
& \Delta y_{i}=y_{i} \otimes t_{i}^{-1}+1 \otimes y_{i}, \quad \sigma\left(y_{i}\right)=-y_{i} t_{i}, \quad \epsilon\left(y_{i}\right)=0, \\
& \Delta t_{i}=t_{i} \otimes t_{i}, \quad \sigma\left(t_{i}\right)=t_{i}^{-1}, \quad \epsilon\left(t_{i}\right)=1 .
\end{aligned}
$$

The algebra $U$ acts on itself from the right by the right adjoint action, denoted by $\operatorname{ad}_{r}$. This action is completely determined by the action of the generators of $U$ as follows:

$$
\begin{aligned}
\left(\operatorname{ad}_{r} t_{i}\right) b & =t_{i}^{-1} b t_{i}, \\
\left(\operatorname{ad}_{r} x_{i}\right) b & =t_{i}^{-1} b x_{i}-t_{i}^{-1} x_{i} b, \\
\left(\operatorname{ad}_{r} y_{i}\right) b & =b y_{i}-y_{i} t_{i} b t_{i}^{-1}
\end{aligned}
$$

for all $b \in U$ and $1 \leq i \leq n$. Throughout this paper we will encounter various right $U$-modules for which the right action is induced by the adjoint action of $U$ on itself. We will frequently call such modules $\left(\operatorname{ad}_{r} U\right)$-modules. Similarly, we will talk of $\left(\operatorname{ad}_{r} M\right)$-modules for subalgebras $M$ of $U$ and its extensions.

Let $U^{+}$denote the subalgebra of $U$ generated by $x_{1}, \ldots, x_{n}$ and let $U^{-}$denote the subalgebra of $U$ generated by $y_{1}, \ldots, y_{n}$. Similarly, let $G^{+}$and $G^{-}$denote the subalgebras of $U$ generated by $x_{1} t_{1}^{-1}, \ldots, x_{n} t_{n}^{-1}$ and $y_{1} t_{1}, \ldots, y_{n} t_{n}$, respectively. Let $T$ denote the multiplicative group generated by $t_{1}^{ \pm 1}, \ldots, t_{n}^{ \pm 1}$ and let $U^{0}$ denote the subalgebra of $U$ generated by $T$. The algebra $U$ admits a triangular decomposition. In particular, the multiplication map induces an isomorphism

$$
U^{-} \otimes U^{0} \otimes U^{+} \rightarrow U
$$

The same holds if one or both of $U^{-}$and $U^{+}$are replaced by $G^{-}$and $G^{+}$, respectively.

Let $\tau$ denote the group isomorphism from $Q(\pi)$ onto $T$ given by $\tau\left(\alpha_{i}\right)=t_{i}$ for $i=1, \ldots, n$. We can enlarge $T$ to a group $\check{T}$ such that $\tau$ extends to an isomorphism from $P(\pi)$ onto $\check{T}$. It is often convenient to consider the simply connected quantized enveloping algebra $\check{U}$ generated by $U$ and $\check{T}$. In particular, $\check{U}$ is generated by $x_{i}, y_{i}$, 
for $i=1, \ldots, n$, and $\tau(\lambda)$, for $\lambda \in P(\pi)$, satisfying all the relations of $U$ and $\check{T}$ as well as

$$
\tau(\lambda) x_{j}=q^{\left(\lambda, \alpha_{j}\right)} x_{j} \tau(\lambda), \quad \tau(\lambda) y_{j}=q^{-\left(\lambda, \alpha_{j}\right)} y_{j} \tau(\lambda)
$$

for all $\lambda \in P(\pi)$ and $1 \leq j \leq n$. The subalgebra of $\check{U}$ generated by the elements $\tau(\lambda), \lambda \in P(\pi)$, is denoted by $\check{U}^{0}$.

The following notation is used throughout this paper. For any multi-index $I=$ $\left(i_{1}, \ldots, i_{m}\right), 1 \leq i_{j} \leq n$, define $(y t)_{I}=y_{i_{1}} t_{i_{1}} \cdots y_{i_{m}} t_{i_{m}}$. Set $\operatorname{wt}(I)=\alpha_{i_{1}}+\cdots+\alpha_{i_{m}}$. Note that $(y t)_{I} \in G^{-}$. Let $\mathcal{I}$ be a set of multi-indices such that $\left\{(y t)_{I} \mid I \in \mathcal{I}\right\}$ is a basis of $G^{-}$.

1.3. Left and right $U$-modules. Let $M$ be a left $U$-module. Given a vector subspace $S$ of $M$ and a weight $\mu \in P(\pi)$, the $\mu$ weight space of $S$ is the subspace $S_{\mu}$ defined by

$$
S_{\mu}=\left\{a \in S \mid t_{i} a=q^{\left(\alpha_{i}, \mu\right)} a \text { for all } i=1, \ldots, n\right\} .
$$

A weight vector $v$ of $S$ is called a highest weight vector provided that $v \neq 0$ and $x_{i} v=0$ for all $1 \leq i \leq n$. Similarly, a lowest weight vector $v$ is a nonzero weight vector with $y_{i} v=0$ for $1 \leq i \leq n$.

Following standard notation as in [Jos95], the weight spaces of $\check{U}$ are weight spaces with respect to the left adjoint action. In particular, given a subset $M$ of $\check{U}$ and a weight $\mu \in P(\pi)$, the subset $M_{\mu}$ is defined by

$$
M_{\mu}=\left\{a \in M \mid \tau(\lambda) a \tau(\lambda)^{-1}=q^{(\lambda, \mu)} a \text { for all } \lambda \in P(\pi)\right\} .
$$

For $\mu \in P^{+}(\pi)$, let $V(\mu)$ denote the uniquely determined finite-dimensional simple left $U$-module of highest weight $\mu$.

To stick to the conventions of [Let02] we will consider left coideal subalgebras of $\check{U}$ in Section 1.5. It follows from Theorem 1.6 (below) that it will be natural to consider right $U$-modules in order to determine the center of these coideal subalgebras. Therefore, we will consider the dual space $V(\mu)^{*}$ always with its natural right $U$ module structure defined by $f u(v)=f(u v)$ for all $f \in V(\mu)^{*}, v \in V(\mu)$, and $u \in U$.

For a subspace of a right $U$-module $M$ one defines the right $\mu$ weight space as in (44) with $t_{i} a$ replaced by $a t_{i}$. Note that the right $U$-module $V(\mu)^{*}$ has a nonzero $\mu$ weight space. However, the elements of this weight space are annihilated by each of the $y_{i}, 1 \leq i \leq n$. Hence we call a nonzero weight vector $v$ of a right $U$-module a highest weight vector if $v y_{i}=0$ for all $i=1, \ldots, n$. It should also be noted that $M_{\mu}$ as defined in (5) is the $-\mu$ weight space for the right adjoint action.

1.4. Classical infinitesimal symmetric pairs. Let $\theta$ be an involutive Lie algebra automorphism of $\mathfrak{g}$. Write $\mathfrak{g}^{\theta}$ for the Lie subalgebra of $\mathfrak{g}$ consisting of elements fixed by $\theta$. The pair $\left(\mathfrak{g}, \mathfrak{g}^{\theta}\right)$ is a (infinitesimal) symmetric pair. A classification of involutions and symmetric pairs up to isomorphism can be found in Hel78, Chapter $\mathrm{X}$, Sections 2, 5, and 6] and [OV94, Section 4.1.4]. Define $\mathfrak{p}=\{x \in \mathfrak{g} \mid \theta(x)=-x\}$, and hence $\mathfrak{g}=\mathfrak{g}^{\theta} \oplus \mathfrak{p}$. We further assume that the involution $\theta$ is maximally split with respect to $\mathfrak{h}$ and the chosen triangular decomposition of $\mathfrak{g}$ in the sense of Let02, Section 7]. This means in particular that $\mathfrak{a}:=\mathfrak{p} \cap \mathfrak{h}$ is equal to its own centralizer in $\mathfrak{p}$. It should be noted that every involution of $\mathfrak{g}$ is conjugate to a maximally split one inside the group of Lie algebra automorphisms of $\mathfrak{g}$. 
The involution $\theta$ induces an involution $\Theta$ of the root system $\Delta$ of $\mathfrak{g}$ and thus an automorphism of $\mathfrak{h}^{*}$. Define $\pi_{\Theta}=\left\{\alpha_{i} \in \pi \mid \Theta\left(\alpha_{i}\right)=\alpha_{i}\right\}$. Let $p$ be the permutation of $\{1, \ldots, n\}$ such that

$$
\Theta\left(\alpha_{i}\right) \in-\alpha_{p(i)}+\mathbb{Z} \pi_{\Theta} \text { for all } \alpha_{i} \notin \pi_{\Theta}
$$

and $p(i)=i$ if $\alpha_{i} \in \pi_{\Theta}$. Moreover, let $\pi^{*}$ be the set of all $\alpha_{i} \in \pi \backslash \pi_{\Theta}$ such that $i=p(i)$ or $i<p(i)$.

There is a second root system associated to the symmetric pair $\left(\mathfrak{g}, \mathfrak{g}^{\theta}\right)$ defined as follows. Given $\alpha \in \mathfrak{h}^{*}$, set $\tilde{\alpha}=(\alpha-\Theta(\alpha)) / 2$. The subset

$$
\Sigma=\{\tilde{\alpha} \mid \alpha \in \Delta \text { and } \Theta(\alpha) \neq \alpha\}
$$

of $\mathfrak{h}^{*}$ is the restricted root system associated to the pair $\left(\mathfrak{g}, \mathfrak{g}^{\theta}\right)$. The set of simple roots of $\Sigma$ is just $\left\{\tilde{\alpha}_{i} \mid \alpha_{i} \in \pi^{*}\right\}$. Let $P(\Sigma)$ denote the weight lattice of $\Sigma$. Let $P^{+}(\Sigma)$ be the set of dominant integral weights with respect to $\Sigma$.

The partial ordering $\leq$ on $\mathfrak{h}^{*}$ restricts to a partial ordering on $P(\Sigma)$. It is also useful to introduce a restricted version of the standard partial ordering. In particular, given $\mu, \gamma \in P(\Sigma)$, we write $\mu \leq_{r} \gamma$ if $\gamma-\mu \in \sum_{\alpha_{i} \in \pi^{*}} \mathbb{N} \tilde{\alpha}_{i}$.

1.5. Quantum symmetric pairs. The main result of [Let02] is the classification for maximally split symmetric pairs $\left(\mathfrak{g}, \mathfrak{g}^{\theta}\right)$ of all left coideal subalgebras $B$ inside $U$ satisfying the following two properties:

- $B$ specializes to $U\left(\mathfrak{g}^{\theta}\right)$ as $q$ goes to 1 .

- If $B \subseteq C$ and $C$ is a subalgebra of $U$ which specializes to $U\left(\mathfrak{g}^{\theta}\right)$, then $B=\bar{C}$.

The pair $U, B$ is called a quantum symmetric pair and is an analog of the pair of enveloping algebras $U(\mathfrak{g}), U\left(\mathfrak{g}^{\theta}\right)$. We briefly review the structure of the coideal subalgebras $B$.

Let $\mathcal{M}$ denote the Hopf subalgebra of $U$ generated by $x_{i}, y_{i}, t_{i}^{ \pm 1}$ for $\alpha_{i} \in \pi_{\Theta}$. Define the subgroup $T_{\Theta}$ of $T$ by

$$
T_{\Theta}=\{\tau(\lambda) \mid \lambda \in Q(\pi) \text { and } \Theta(\lambda)=\lambda\} .
$$

The subalgebra $B$ of $U$ is generated by $\mathcal{M}, T_{\Theta}$, and elements $B_{i}$ for $\alpha_{i} \in \pi \backslash \pi_{\Theta}$. For the explicit form of the elements $B_{i}$ consult [Let02, Section 7]. In this paper we only use that

$$
B_{i}=y_{i} t_{i}+d_{i} \tilde{\theta}\left(y_{i}\right) t_{i}+s_{i} t_{i}
$$

where $d_{i}, s_{i} \in \mathcal{C}, \tilde{\theta}\left(y_{i}\right) \in G_{\Theta\left(-\alpha_{i}\right)}^{+}$, and $\tilde{\theta}\left(y_{i}\right) t_{i} \in \mathcal{M} x_{p(i)} T_{\Theta}$. It is shown in Let02 that any maximal left coideal subalgebra of $U$ that specializes to $U\left(\mathfrak{g}^{\theta}\right)$ as $q$ goes to 1 is isomorphic to an algebra $B$ of the above form via a Hopf algebra automorphism of $U$.

For the convenience of the reader we recall the relation between the constituents of $B$ and those of $U\left(\mathfrak{g}^{\theta}\right)$ along the lines of [Let99b, Section 3]. Recall that $\mathfrak{a}=\mathfrak{p} \cap \mathfrak{h}$ and let $\mathfrak{m}_{\Theta}$ denote the Lie subalgebra of $\mathfrak{g}^{\theta}$ which centralizes $\mathfrak{a}$. Let $\mathfrak{m}$ denote the semisimple part of $\mathfrak{m}_{\Theta}$. Then $\mathcal{M}$ and $\mathcal{M} T_{\Theta}$ are quantum analogs of the universal enveloping algebras $U(\mathfrak{m})$ and $U\left(\mathfrak{m}_{\Theta}\right)$, respectively. Moreover, the generators $B_{i}$, $\alpha_{i} \in \pi^{*}$, are quantum analogs of the elements $f_{\alpha_{i}}+\theta\left(f_{\alpha_{i}}\right) \in \mathfrak{g}^{\theta}$ where $f_{\alpha_{i}}$ is a nonzero element of the root space $\mathfrak{g}_{-\alpha_{i}}$. 
Set $B_{i}=y_{i} t_{i}$ for $\alpha_{i} \in \pi_{\Theta}$ and set $\mathcal{M}^{+}=\mathcal{M} \cap U^{+}$. Recall the definition of $\mathcal{I}$ given in Section 1.2. Given a multi-index $I=\left(i_{1}, \ldots, i_{m}\right)$ in $\mathcal{I}$, set $B_{I}=B_{i_{1}} \cdots B_{i_{m}}$. By [Let02, Section 7], we have the direct sum decomposition

$$
B=\bigoplus_{I \in \mathcal{I}} B_{I} \mathcal{M}^{+} T_{\Theta}
$$

It follows that when $b \in B$ is written as a sum of weight vectors in $\check{U}$, any lowest weight term is an element of $G^{-} \mathcal{M}^{+} T_{\Theta}$.

Let $T_{\Theta}^{\prime}$ be the subalgebra of $\check{U}$ defined by

$$
T_{\Theta}^{\prime}=\{\tau(\lambda) \mid \lambda \in P(\pi) \text { and } \Theta(\lambda)=\lambda\} .
$$

Let $\check{B}$ be the subalgebra of $\check{U}$ generated by $B$ and $T_{\Theta}^{\prime}$. It follows from properties of $B$ that $\check{B}$ is a left coideal subalgebra of $\check{U}$ that specializes to $U\left(\mathfrak{g}^{\theta}\right)$ as $q$ goes to 1 . It should be noted that the direct sum decomposition (8) as well as the subsequent remark also hold for $\check{B}$ if $T_{\Theta}$ is replaced by the group $T_{\Theta}^{\prime}$.

1.6. The locally finite part of $\check{U}$. The locally finite part of $\check{U}$ with respect to the right adjoint action of $U$ on $\check{U}$ is the set

$$
F_{r}(\check{U})=\left\{a \in \check{U} \mid \operatorname{dim}\left(\left(\operatorname{ad}_{r} U\right) a\right)<\infty\right\} .
$$

We recall here some basic facts about $F_{r}(\check{U})$ from [JL94 (see also [Jos95]). However, it should be noted that JL94 is written in terms of the left adjoint action while here we focus on the right adjoint action. Definitions and theorems from [JL94] will be translated accordingly.

The vector space $F_{r}(\check{U})$ is a subalgebra of $\check{U}$. As an $\left(\operatorname{ad}_{r} U\right)$-module, it can be decomposed as follows [JL94, Thm. 4.10]

$$
F_{r}(\check{U})=\bigoplus_{\mu \in P^{+}(\pi)}\left(\operatorname{ad}_{r} U\right) \tau(2 \mu)
$$

Recall further the isomorphism $\left(\operatorname{ad}_{r} U\right) \tau(2 \mu) \cong V(\mu)^{*} \otimes V\left(-w_{0} \mu\right)^{*}$ of right $U$ modules [JL94, Corollary 3.5].

Consider $\mu \in P^{+}(\pi)$. The following formula is useful in analyzing the $\left(\operatorname{ad}_{r} U\right)$ module generated by $\tau(2 \mu)$. Let $\zeta$ and $\xi$ be elements in $Q^{+}(\pi)$. The description of the right adjoint action combined with the relations of $U$ ensure that

$$
\left(\operatorname{ad}_{r} U_{-\zeta}^{-} U_{\xi}^{+}\right) \tau(2 \mu) \in \sum_{\alpha \leq \zeta} \sum_{\beta \leq \xi} \sum_{\substack{0 \leq \eta \leq \zeta-\alpha \\ 0 \leq \eta \leq \xi-\beta}} U_{-\alpha}^{-} G_{\beta}^{+} \tau(2 \mu-2 \eta) .
$$

Let $Z(\check{B})$ denote the center of $\check{B}$. The fact that $\check{B}$ is a left coideal implies that any element in $Z(\check{B})$ spans a one-dimensional $\left(\operatorname{ad}_{r} B\right)$-invariant submodule of $\check{U}$. The following observation will allow us to use the well known structure of the locally finite part $F_{r}(\check{U})$ in the investigation of the center of $\check{B}$.

Theorem 1.6 ([Let08, Theorem 1.2]). Each finite-dimensional $\left(\operatorname{ad}_{r} B\right)$-submodule of $\check{U}$ is a subset of $F_{r}(\check{U})$. In particular, $Z(\check{B})$ is a subalgebra of $F_{r}(\check{U})$. 
1.7. Spherical modules. Given a $U$-module $M$, let $M^{B}$ denote the set of $B$ invariant elements inside of $M$. For example, if $M$ is a left $U$-module, then $M^{B}=$ $\{v \in M \mid b v=\varepsilon(b) v$ for all $b \in M\}$. For any finite-dimensional simple $U$-module $V(\lambda), \lambda \in P^{+}(\pi)$, one has $\operatorname{dim}\left(V(\lambda)^{B}\right) \leq 1$ Let02, Theorem 7.7], and $V(\lambda)$ is called spherical if $V(\lambda)^{B}$ is one-dimensional. It is shown in Let03, Theorem 3.4] that $V(\lambda)$ is a finite-dimensional spherical $U$-module if and only if $\lambda \in 2 P^{+}(\Sigma)$. If $\lambda \in 2 P^{+}(\Sigma)$, then we refer to $\lambda$ as a spherical weight.

Fix $\lambda \in P^{+}(\pi)$ and let $v_{\lambda}$ denote the highest weight vector of $V(\lambda)$. Assume further that $V(\lambda)$ is spherical and let $v^{B}$ be a nonzero $B$-invariant element of $V(\lambda)$. Rescaling if necessary, we can write

$$
v^{B}=v_{\lambda}+\sum_{\left\{\mu \in P(\Sigma) \mid \mu<_{r} \lambda\right\}} w_{\mu}
$$

where each $w_{\mu}$ is a vector of weight $\mu$ inside $V(\lambda)$ [Let03, Theorem 3.6].

By [Let02, Theorem 7.6] the left $B$-module $V(\lambda)$ is a direct sum of irreducible left $B$-modules. Hence the right $B$-module $V(\lambda)^{*}$ also decomposes into irreducible right $B$-modules. Thus $V(\lambda)^{*}$ contains a right $B$-invariant element if and only if $V(\lambda)$ contains a left $B$-invariant element. Moreover, this holds if and only if $\lambda$ is spherical.

\section{Two $\left(\operatorname{ad}_{r} U\right)$-INVARIANT FILTRATIONS}

In this section, we consider two $\left(\operatorname{ad}_{r} U\right)$-invariant filtrations on $\check{U}$. The first filtration $\mathcal{F}$ is one of the basic tools used to analyze the locally finite part of $\check{U}$ in JJL94 (see also Jos95, Chapter 7]). The second filtration $\mathcal{F}^{\theta}$ is a modification of $\mathcal{F}$ that is based on the restricted root system $\Sigma$ in the same way as $\mathcal{F}$ is based on the root system $\pi$. An isomorphism of the locally finite parts of the graded algebras of $\check{U}$ with respect to these two different filtrations is established at the end of the section.

2.1. The standard $\left(\operatorname{ad}_{r} U\right)$-invariant filtration. Let $h: \mathbb{Q} \pi \rightarrow \mathbb{Q}$ denote the height function defined by

$$
h\left(\sum_{i} n_{i} \alpha_{i}\right)=\sum_{i} n_{i} .
$$

Note that the image of $P(\pi)$ under the height function is contained in $\frac{1}{N} \mathbb{Z}$ for some sufficiently large positive integer $N$. Consider the $\frac{1}{N} \mathbb{Z}$-filtration $\mathcal{F}$ of $\tilde{U}$ defined by the following degree function

$$
\operatorname{deg}\left(x_{i} t_{i}^{-1}\right)=\operatorname{deg}\left(y_{i}\right)=0, \quad \operatorname{deg} \tau(\lambda)=h(\lambda)
$$

for all $i=1, \ldots, n$ and $\lambda \in P(\pi)$. Note that $\mathcal{F}$ restricts to a $\mathbb{Z}$-filtration on $U$. Define $\mathrm{Gr}:=\operatorname{gr}_{\mathcal{F}}(\check{U}), \mathrm{Gr}^{0}:=\operatorname{gr}_{\mathcal{F}}\left(\check{U}^{0}\right), \mathrm{Gr}^{-}:=\operatorname{gr}_{\mathcal{F}}\left(U^{-}\right)$, and $\mathrm{Gr}^{+}:=\operatorname{gr}_{\mathcal{F}}\left(G^{+}\right)$to be the associated graded algebras. The description (1) of the right adjoint action of $U$ on $\check{U}$ implies that $\mathcal{F}$ is an $\left(\operatorname{ad}_{r} U\right)$-invariant filtration. In particular, Gr inherits the structure of an $\left(\operatorname{ad}_{r} U\right)$-module from $\check{U}$. Given $a \in \check{U}$, we denote its graded image in Gr by $\bar{a}$. More precisely, for each $a \in \mathcal{F}_{n}(\check{U}) \backslash \mathcal{F}_{n-1 / N}(\check{U})$ we write $\bar{a}$ for the image of $a$ in the subspace $\mathcal{F}_{n}(\check{U}) / \mathcal{F}_{n-1 / N}(\check{U})$ of Gr.

Note that $U^{-} \subseteq \mathcal{F}_{0} U^{-}$and $\mathcal{F}_{-1 / N} U^{-}=\{0\}$. Hence $U^{-} \cong \mathrm{Gr}^{-} \cong \mathrm{Gr}_{0}^{-}$. Similarly, one obtains $G^{+} \cong \mathrm{Gr}^{+} \cong \mathrm{Gr}_{0}^{+}$. Note that $\mathrm{Gr}^{0}, \mathrm{Gr}^{-}$, and $\mathrm{Gr}^{+}$are 
subalgebras of Gr. By the triangular decomposition (2) the multiplication map

$$
\mathrm{Gr}^{-} \otimes \mathrm{Gr}^{0} \otimes \mathrm{Gr}^{+} \rightarrow \mathrm{Gr}
$$

is an isomorphism. Using this isomorphism, we may identify $\mathrm{Gr}^{-} \otimes \mathrm{Gr}^{0}$ with $\mathrm{Gr}^{-} \mathrm{Gr}^{0}$ and $\mathrm{Gr}^{0} \otimes \mathrm{Gr}^{+}$with $\mathrm{Gr}^{0} \mathrm{Gr}^{+}$as vector spaces.

2.2. The locally finite part of Gr. Given $\mu \in P^{+}(\pi)$, define the subspace $K(2 \mu)^{+}$of $\mathrm{Gr}^{+}$and the subspace $K(2 \mu)^{-}$of $\mathrm{Gr}^{-}$by

$$
\begin{aligned}
& \left(\operatorname{ad}_{r} U^{-}\right) \overline{\tau(2 \mu)}=K(2 \mu)^{-} \otimes \mathcal{C} \overline{\tau(2 \mu)} \\
& \left(\operatorname{ad}_{r} U^{+}\right) \overline{\tau(2 \mu)}=\mathcal{C} \overline{\tau(2 \mu)} \otimes K(2 \mu)^{+} .
\end{aligned}
$$

By [JL94, Section 4.9] one has

$$
\left(\operatorname{ad}_{r} U\right) \overline{\tau(2 \mu)}=K(2 \mu)^{-} \otimes \mathcal{C} \overline{\tau(2 \mu)} \otimes K(2 \mu)^{+} .
$$

Lemma 2.2. Given $\mu \in P^{+}(\pi)$ and $G \in K(2 \mu)_{\alpha}^{-} \otimes \mathcal{C} \overline{\tau(2 \mu)} \otimes K(2 \mu)_{-\beta}^{+}$, there exists an element $a \in \sum_{\zeta \leq \alpha} \sum_{\xi \leq \beta} U_{-\zeta}^{-} U_{\xi}^{+}$such that $\left(\operatorname{ad}_{r} a\right) \overline{\tau(2 \mu)}=G$.

Proof. Without loss of generality, we may assume that $G=u^{\prime} \otimes \overline{\tau(2 \mu)} \otimes v^{\prime}$ for some $u^{\prime} \in K(2 \mu)_{\alpha}^{-}$and $v^{\prime} \in K(2 \mu)_{-\beta}^{+}$. By (14) and (15) there exist $u \in U^{-}$and $v \in U^{+}$ such that $\left(\operatorname{ad}_{r} u\right) \tau(2 \mu)=u^{\prime} \otimes \overline{\tau(2 \mu)}$ and $\left(\operatorname{ad}_{r} v\right)=\overline{\tau(2 \mu)} \otimes v^{\prime}$. We may further assume that $u \in U_{-\alpha}^{-}$and $v \in U_{\beta}^{+}$because the adjoint action on $\overline{\tau(2 \mu)}$ preserves weight spaces. Using the fact that $K(2 \mu)^{-}$is an $\left(\operatorname{ad}_{r} U^{+}\right)$-submodule of $\mathrm{Gr}^{-}$[JL94, Section 4.9] combined with the relations of $U$ one obtains

$$
\begin{aligned}
& \left(\operatorname{ad}_{r} u v\right) \overline{\tau(2 \mu)}=\left(\operatorname{ad}_{r} v\right)\left(u^{\prime} \otimes \overline{\tau(2 \mu)}\right) \\
& \quad \in q^{(\alpha, \beta)} u^{\prime} \otimes \overline{\tau(2 \mu)} \otimes v^{\prime}+\sum_{\substack{\eta<\alpha \\
\xi<\beta}} K(2 \mu)_{\eta}^{-} \otimes \overline{\tau(2 \mu)} \otimes K(2 \mu)_{-\xi}^{+} .
\end{aligned}
$$

The lemma follows by induction on the height of $\alpha$ and $\beta$.

By [Jos95, Lemma 7.1.4] the $\left(\operatorname{ad}_{r} U\right)$-modules $\left(\operatorname{ad}_{r} U\right) \overline{\tau(2 \mu)}$ and $\left(\operatorname{ad}_{r} U\right) \tau(2 \mu)$ are isomorphic for each $\mu \in P^{+}(\pi)$. Moreover, the locally finite part $F_{r}(\mathrm{Gr})$ of $\mathrm{Gr}$ is isomorphic to $F_{r}(\check{U})$ as an $\left(\operatorname{ad}_{r} U\right)$-module [JL94, Sec. 4.10], [Jos95, Sec. 7.1]. This isomorphism is given by the function $g: F_{r}(\check{U}) \rightarrow F_{r}(\mathrm{Gr})$ defined by

$$
g\left(\left(\operatorname{ad}_{r} u\right) \tau(2 \mu)\right)=\left(\operatorname{ad}_{r} u\right) \overline{\tau(2 \mu)}
$$

for all $\mu \in P^{+}(\pi)$ and $u \in U$. The isomorphism $g$ combined with the decomposition of $F_{r}(\check{U})$ in (9) yields the following direct sum decomposition:

$$
F_{r}(\mathrm{Gr})=\bigoplus_{\mu \in P^{+}(\pi)}\left(\operatorname{ad}_{r} U\right) \overline{\tau(2 \mu)}
$$

2.3. A coarse $\operatorname{ad}_{r}$-invariant filtration. The second filtration is defined using a height function associated to the restricted root system instead of the ordinary height function. More precisely, the height function $h^{\Sigma}$ associated to the restricted root system is the function $h^{\Sigma}: \mathbb{Q} \Sigma \rightarrow \mathbb{Q}$ defined by

$$
h^{\Sigma}\left(\sum_{\alpha_{i} \in \pi^{*}} n_{i} \tilde{\alpha}_{i}\right)=\sum_{\alpha_{i} \in \pi^{*}} n_{i} .
$$


The function $h^{\Sigma}$ can be extended to a function on $\mathbb{Q} \pi$ by setting $h^{\Sigma}(\lambda)=h^{\Sigma}(\tilde{\lambda})$ for all $\lambda \in \mathbb{Q} \pi$. Note that $h^{\Sigma}$ restricts to a function from $P(\pi)$ to $\frac{1}{N} \mathbb{Z}$ for a sufficiently large positive integer $N$. For the remainder of this paper, we assume that $N$ has been chosen so that both height functions $h$ and $h^{\Sigma}$ map $P(\pi)$ to $\frac{1}{N} \mathbb{Z}$.

Define a $\frac{1}{N} \mathbb{Z}$-filtration $\mathcal{F}^{\theta}$ of $\check{U}$ by the degree function

$$
\operatorname{deg}\left(x_{i} t_{i}^{-1}\right)=\operatorname{deg}\left(y_{i}\right)=0, \quad \operatorname{deg}(\tau(\lambda))=h^{\Sigma}(\lambda)
$$

for all $i=1, \ldots, n$ and $\lambda \in P(\pi)$. Note that $\mathcal{F}^{\theta}$ restricts to a $\mathbb{Z}$-filtration on $U$. Moreover, $\mathcal{F}^{\theta}$ is an $\left(\operatorname{ad}_{r} U\right)$-invariant on both $U$ and $\check{U}$. The analogous filtration invariant under the left adjoint action has been used in Let04, Sec. 5]. Let $\mathrm{Gr}^{\theta}=$ $\operatorname{gr}_{\mathcal{F}^{\theta}}(\check{U})$ be the associated graded algebra. Given $a \in \check{U}$, we denote its graded image in $\mathrm{Gr}^{\theta}$ by $\bar{a}^{\theta}$.

Consider weights $\alpha$ and $\beta$ in $Q(\pi)$ such that $\alpha \leq \beta$. Note that if $h^{\Sigma}(\alpha)<h^{\Sigma}(\beta)$, then $\alpha<\beta$ and so $h(\alpha)<h(\beta)$. This observation will be useful in comparing the two filtrations $\mathcal{F}$ and $\mathcal{F}^{\theta}$.

2.4. The locally finite part of $\mathrm{Gr}^{\theta}$. The locally finite part of $\mathrm{Gr}^{\theta}$ with respect to the right adjoint action of $U$ on $\check{U}$ is the set

$$
F_{r}\left(\operatorname{Gr}^{\theta}\right)=\left\{a \in \operatorname{Gr}^{\theta} \mid \operatorname{dim}\left(\left(\operatorname{ad}_{r} U\right) a\right)<\infty\right\} .
$$

An immediate consequence of the following result is that the $\frac{1}{N} \mathbb{Z}$-graded $\left(\operatorname{ad}_{r} U\right)$ module $F_{r}\left(\mathrm{Gr}^{\theta}\right)$ can be obtained from the $\frac{1}{N} \mathbb{Z}$-graded $\left(\operatorname{ad}_{r} U\right)$-module $F_{r}(\mathrm{Gr})$ by renumbering degrees of the submodules of the form $\left(\operatorname{ad}_{r} U\right) \overline{\tau(2 \mu)}$.

Proposition 2.4. The map $g^{\theta}: F_{r}(\check{U}) \rightarrow F_{r}\left(\mathrm{Gr}^{\theta}\right)$ defined by

$$
g^{\theta}\left(\left(\operatorname{ad}_{r} u\right) \tau(2 \mu)\right)=\left(\operatorname{ad}_{r} u\right) \overline{\tau(2 \mu)}^{\theta}
$$

for all $\mu \in P^{+}(\pi)$ and $u \in U$ is an isomorphism of right $U$-modules.

Proof. Recall that $F_{r}(\check{U})$ and $F_{r}(\mathrm{Gr})$ are isomorphic as $\left(\operatorname{ad}_{r} U\right)$-modules via the map $g$ defined in (17). Note that by the discussion at the end of Section 2.3, one gets ker $g^{\theta} \subseteq \operatorname{ker} g$. Therefore, $g^{\theta}$ is injective.

To prove surjectivity let $v \in \mathcal{F}_{s}^{\theta} \check{U}$ represent an element $\bar{v}^{\theta} \in F_{r}\left(\operatorname{Gr}_{s}^{\theta}\right)$. In particular, $\operatorname{dim}\left(\left(\operatorname{ad}_{r} U\right) \bar{v}^{\theta}\right)<\infty$. Without loss of generality, we may assume that there exist $\lambda_{1}, \ldots, \lambda_{k}$ such that $h^{\Sigma}\left(\lambda_{i}\right)=s$ for all $i=1, \ldots, k$ and

$$
v \in \sum_{i=1}^{k} U^{-} \tau\left(\lambda_{i}\right) G^{+} .
$$

It follows from the description of the right adjoint action (11) and the defining relations of $U$ that

$$
\left(\operatorname{ad}_{r} U\right) v \in \sum_{i=1}^{k} \sum_{\mu \leq \lambda_{i}} U^{-} \tau(\mu) G^{+} .
$$

Since $\left(\operatorname{ad}_{r} U\right) \bar{v}^{\theta}$ is finite dimensional, there exist subspaces $U_{i}$ of $U^{-}$and $G_{i}$ of $G^{+}$ such that

$$
\left(\operatorname{ad}_{r} U\right) v \subseteq \sum_{i=1}^{k} U_{i} \tau\left(\lambda_{i}\right) G_{i}+\sum_{i=1}^{k} \sum_{\mu<\lambda_{i}} U^{-} \tau(\mu) G^{+}
$$


Let $m$ be the smallest rational number such that $v \in \mathcal{F}_{m}(\check{U})$. It follows that

$$
\left(\operatorname{ad}_{r} U\right) v \subseteq \sum_{\left\{i \mid h\left(\lambda_{i}\right)=m\right\}} U_{i} \tau\left(\lambda_{i}\right) G_{i}+\sum_{\{\mu \in P(\pi) \mid h(\mu)<m\}} U^{-} \tau(\mu) G^{+} .
$$

Note that (21) ensures that $\left(\operatorname{ad}_{r} U\right) \bar{v}$ is contained in the image of the finite-dimensional subspace $\sum_{\left\{i \mid h\left(\lambda_{i}\right)=m\right\}} U_{i} \tau\left(\lambda_{i}\right) G_{i}$ inside Gr. In particular, $\bar{v} \in F_{r}(\mathrm{Gr})$. It follows from (18) that

$$
v \in \sum_{\left\{i \mid h\left(\lambda_{i}\right)=m\right\}}\left(\operatorname{ad}_{r} U\right) \tau\left(\lambda_{i}\right)+\sum_{\{\mu \in P(\pi) \mid h(\mu)<m\}} U^{-} \tau(\mu) G^{+} .
$$

Moreover, $\lambda_{i} \in 2 P^{+}(\pi)$ for all $i$ such that $h\left(\lambda_{i}\right)=m$. In particular, $m$ is a nonnegative element of $\frac{1}{N} \mathbb{Z}$.

Using (22), we can choose $w \in \sum_{h\left(\lambda_{i}\right)=m}\left(\operatorname{ad}_{r} U\right) \tau\left(\lambda_{i}\right)$ such that $v-w$ is of smaller filter degree than $m$ with respect to $\mathcal{F}$. Since each $\lambda_{i} \in 2 P^{+}(\pi)$, we see that $\bar{w}^{\theta}$ is in $F_{r}\left(\mathrm{Gr}^{\theta}\right)$. Assume first that $m=0$. Suppose that $\bar{v}^{\theta} \neq \bar{w}^{\theta}$. It follows that $\bar{v}^{\theta}-\bar{w}^{\theta}=\overline{(v-w)}^{\theta}$. Hence, $\overline{(v-w)}^{\theta}$ is an element of $F_{r}\left(\operatorname{Gr}^{\theta}\right)$. Arguing as above with $v$ replaced by $v-w$, we see that $v-w \in \mathcal{F}_{c}(\check{U})$ for some nonnegative element $c$ of $\frac{1}{N} \mathbb{Z}$. Moreover, $c$ must be strictly less than $m$ and $m=0$. This contradiction forces $\bar{v}^{\theta}=\bar{w}^{\theta}$. Therefore, $\bar{v}^{\theta}=g^{\theta}(w)$ and so $\bar{v}^{\theta}$ is in the image of $g^{\theta}$. Now assume that $m>0$. If $\bar{v}^{\theta}=\bar{w}^{\theta}$, then once again $\bar{v}^{\theta}$ is in the image of $g^{\theta}$. Thus we may assume that $\bar{v}^{\theta} \neq \bar{w}^{\theta}$. Replacing $v$ by $v-w$, we obtain $v-w \in \mathcal{F}_{c}(\check{U})$ for some nonnegative element $c$ of $\frac{1}{N} \mathbb{Z}$ strictly less than $m$. By induction, $\overline{v-w}^{\theta} \in g^{\theta}\left(F_{r}(\check{U})\right)$. The lemma now follows from the fact that $\bar{w}^{\theta}$ is in the image of $g^{\theta}$ and $\bar{v}^{\theta}-\bar{w}^{\theta}=\overline{v-w}^{\theta}$.

2.5. B-invariant elements of $\mathrm{Gr}$ and $\mathrm{Gr}^{\theta}$. Recall that $\check{U}$ is semisimple as an $\left(\operatorname{ad}_{r} T\right)$-module. Hence both $\mathrm{Gr}$ and $\operatorname{Gr}^{\theta}$ are semisimple $\left(\operatorname{ad}_{r} T\right)$-modules. It follows from [Let99a, Lemma 3.4] that any element $b \in \mathrm{Gr}^{B}$ generates a finite-dimensional $\left(\operatorname{ad}_{r} U\right)$-submodule. Thus $\mathrm{Gr}^{B} \subseteq F_{r}(\mathrm{Gr})$. Similarly, one has $\left(\mathrm{Gr}^{\theta}\right)^{B} \subseteq F_{r}\left(\mathrm{Gr}^{\theta}\right)$. The second part of the following result is an immediate consequence of Theorem 1.6. Proposition 2.4, and the fact that the map $g$ defined by (9) is an isomorphism.

Theorem 2.5. We have the following inclusions of B-modules:

$$
\mathrm{Gr}^{B} \subseteq F_{r}(\mathrm{Gr}), \quad\left(\mathrm{Gr}^{\theta}\right)^{B} \subseteq F_{r}\left(\mathrm{Gr}^{\theta}\right) .
$$

Moreover, the map $g$ restricts to an isomorphism of $\check{U}^{B}$ onto $\mathrm{Gr}^{B}$ and the map $g^{\theta}$ restricts to an isomorphism of $\check{U}^{B}$ onto $\left(\mathrm{Gr}^{\theta}\right)^{B}$.

\section{Spherical Submodules of $\left(\operatorname{ad}_{r} U\right) \tau(2 \mu)$}

The first lemma of this section describes a property satisfied by highest weight vectors inside submodules of the form $\left(\operatorname{ad}_{r} U\right) \tau(2 \mu)$. We then establish a multiplicity result concerning certain spherical finite-dimensional simple $U$-modules inside $\left(\operatorname{ad}_{r} U\right) \tau(2 \mu)$, for $\mu \in P^{+}(\pi)$. This latter result will help us determine which submodules $\left(\operatorname{ad}_{r} U\right) \tau(2 \mu)$ of $F_{r}(\check{U})$ contain nonzero elements of $Z(\check{B})$ up to lower degree terms with respect to the filtration $\mathcal{F}^{\theta}$. 
3.1. Highest weight vectors of Gr. By [JL94, 4.8], $\left(\operatorname{ad}_{r} U^{-}\right) \overline{\tau(2 \mu)}$ contains a unique one-dimensional highest weight space with respect to the right adjoint action. Moreover, the weight of this space is $\mu-w_{0} \mu$ and all other weights of $\left(\operatorname{ad}_{r} U^{-}\right) \overline{\tau(2 \mu)}$ are strictly smaller than $\mu-w_{0} \mu$. The next lemma provides a useful property of highest weight vectors in Gr.

Lemma 3.1. Let $\mu \in P^{+}(\pi)$ and let $v$ be a highest weight vector in $\left(\operatorname{ad}_{r} U\right) \overline{\tau(2 \mu)}$. Then there exist nonzero weight vectors $X \in K(2 \mu)^{+}$and $Y \in K(2 \mu)_{\mu-w_{0} \mu}^{-}$such that

$$
v \in Y \otimes \overline{\tau(2 \mu)} \otimes X+\sum_{\eta<\mu-w_{0} \mu} K(2 \mu)_{\eta}^{-} \otimes \mathcal{\mathcal { C }} \overline{\tau(2 \mu)} \otimes K(2 \mu)^{+} .
$$

Proof. We can write $v=\sum_{i=1}^{k} Y_{i} \otimes X_{i}$ where each $Y_{i}$ is a weight vector of $K(2 \mu)^{-} \otimes$ $\overline{\tau(2 \mu)}$ and each $X_{i}$ is a weight vector of $K(2 \mu)^{+}$with respect to the right adjoint action. Assume further that $\left\{X_{i} \mid 1 \leq i \leq k\right\}$ is a linearly independent set. Let $\lambda$ denote the weight of $v$ and let $\eta_{i}$ denote the weight of $Y_{i}$. Choose $s \in\{1, \ldots, k\}$ such that $\eta_{s}$ is maximal. Then for all $j=1, \ldots, n$ one has

$$
\begin{aligned}
0 & =\left(\operatorname{ad}_{r} y_{j}\right) v \\
& =\sum_{i=1}^{k}\left(\operatorname{ad}_{r} y_{j}\right) Y_{i} \otimes\left(\operatorname{ad}_{r} t_{i}^{-1}\right) X_{i}+\sum_{i=1}^{k} Y_{i} \otimes\left(\operatorname{ad}_{r} y_{i}\right) X_{i} \\
& \in \sum_{\eta_{i}=\eta_{s}}\left(\operatorname{ad}_{r} y_{j}\right) Y_{i} \otimes\left(\operatorname{ad}_{r} t_{i}^{-1}\right) X_{i}+\sum_{\eta \neq \eta_{s}} K(2 \mu)^{-} \otimes \mathcal{C} \overline{\tau(2 \mu)} \otimes K(2 \mu)_{\lambda-\eta}^{+}
\end{aligned}
$$

and hence $\left(\operatorname{ad}_{r} y_{j}\right) Y_{i}=0$ for all $j=1, \ldots, n$ and for all $i$ such that $\eta_{i}=\eta_{s}$. In particular, $Y_{i}$ is a highest weight vector in $\left(\operatorname{ad}_{r} U^{-}\right) \overline{\tau(2 \mu)}$ whenever $\eta_{i}=\eta_{s}$. The result now follows from the discussion preceding the lemma.

3.2. Multiplicity of spherical modules. Recall that $\pi_{\Theta}$ is the subset of $\pi$ consisting of those simple roots $\alpha_{i}$ such that $\Theta\left(\alpha_{i}\right)=\alpha_{i}$. Write $W\left(\pi_{\Theta}\right)$ for the subgroup of the Weyl group $W$ generated by the simple reflections corresponding to the simple roots in $\pi_{\Theta}$. Note that $W\left(\pi_{\Theta}\right)$ is the Weyl group corresponding to the root system generated by $\pi_{\Theta}$. Let $w_{0}^{\prime}$ denote the longest element in $W\left(\pi_{\Theta}\right)$.

Let $\mathfrak{m}$ denote the semisimple Lie subalgebra of $\mathfrak{g}$ generated by the positive and negative root vectors corresponding to the simple roots in $\pi_{\Theta}$. Note that $\mathcal{M}$ can be identified with the quantized enveloping algebra of $\mathfrak{m}$. Suppose that $\mu \in P^{+}(\pi)$. Then $\mu$ restricts to a dominant integral weight with respect to the root system generated by $\pi_{\Theta}$. It follows as in the discussion preceding Lemma 3.1 that the subspace of $\left(\operatorname{ad}_{r} \mathcal{M}^{+}\right) \overline{\tau(2 \mu)}$ annihilated by each $\left(\operatorname{ad}_{r} x_{i}\right)$ for $\alpha_{i} \in \pi_{\Theta}$ is one dimensional. Moreover, this space is a weight space of weight $-\mu+w_{0}^{\prime} \mu$ with respect to the right adjoint action of $U$.

Theorem 3.2. Let $\lambda$ be a spherical weight and let $\mu \in P^{+}(\pi)$ such that $\mu-w_{0} \mu-\lambda \in$ $\mathbb{N} \pi_{\Theta}$. Then

$$
\left[\left(\operatorname{ad}_{r} U\right) \tau(2 \mu): V(\lambda)^{*}\right] \leq 1 .
$$

Moreover,

$$
\left[\left(\operatorname{ad}_{r} U\right) \tau(2 \mu): V(\lambda)^{*}\right]=1
$$

if and only if $\lambda=w_{0}^{\prime} \mu-w_{0} \mu$. 
Proof. As noted in Section 2.2, the right $U$-modules $\left(\operatorname{ad}_{r} U\right) \overline{\tau(2 \mu)}$ and $\left(\operatorname{ad}_{r} U\right) \tau(2 \mu)$ are isomorphic. Thus it is sufficient to prove (23) and (24) using $\left(\operatorname{ad}_{r} U\right) \overline{\tau(2 \mu)}$ instead of $\left(\operatorname{ad}_{r} U\right) \tau(2 \mu)$. Let $Y_{\lambda}$ denote a highest weight vector of weight $\lambda$ inside $\left(\operatorname{ad}_{r} U\right) \overline{\tau(2 \mu)}$. By the discussion preceding Lemma 3.1, we can choose a unique (up to scalar multiplication) nonzero $Y \in K(2 \mu)^{-}$such that $Y \otimes \overline{\tau(2 \mu)}$ is a highest weight vector of weight $\mu-w_{0} \mu$ in $\left(\operatorname{ad}_{r} U^{-}\right) \overline{\tau(2 \mu)}$. Lemma 3.1 ensures that there exists a nonzero $X \in K(2 \mu)^{+}$such that

$$
Y_{\lambda} \in Y \otimes \overline{\tau(2 \mu)} \otimes X+\sum_{0 \leq \eta<\mu-w_{0} \mu} K(2 \mu)_{\eta}^{-} \otimes \overline{\tau(2 \mu)} \otimes K(2 \mu)_{\lambda-\eta}^{+} .
$$

We claim that

$$
\left(\operatorname{ad}_{r} x_{i}\right)(\overline{\tau(2 \mu)} \otimes X)=0 \text { for all } \alpha_{i} \in \pi_{\Theta} .
$$

Indeed, recall that $\lambda$ is spherical and $Y_{\lambda}$ is a highest weight vector of weight $\lambda$. It follows that $Y_{\lambda}$ is $\left(\operatorname{ad}_{r} \mathcal{M} T_{\Theta}\right)$-invariant. Hence, for $\alpha_{i} \in \pi_{\Theta}$ one has

$$
\begin{aligned}
0=\left(\operatorname{ad}_{r} x_{i}\right) Y_{\lambda} \in\left(\left(\operatorname{ad}_{r} t_{i}\right) Y\right) & \otimes\left(\left(\operatorname{ad}_{r} x_{i}\right)(\overline{\tau(2 \mu)} \otimes X)\right) \\
+ & \sum_{0 \leq \eta<\mu-w_{0} \mu} K(2 \mu)_{\eta}^{-} \otimes \overline{\tau(2 \mu)} \otimes K(2 \mu)^{+}
\end{aligned}
$$

which proves (25).

Note that $Y \otimes \overline{\tau(2 \mu)} \otimes X$ is a vector of weight $\lambda$. It follows that

$$
\overline{\tau(2 \mu)} \otimes X \in \overline{\tau(2 \mu)} \otimes K(2 \mu)_{-\beta}^{+}
$$

where $\beta=\mu-w_{0} \mu-\lambda$. By assumption, $\beta \in \mathbb{N} \pi_{\Theta}$. Hence $\overline{\tau(2 \mu)} \otimes X \in\left(\operatorname{ad}_{r} \mathcal{M}^{+}\right) \overline{\tau(2 \mu)}$. By the discussion preceding the lemma, $\overline{\tau(2 \mu)} \otimes X$ spans the unique one-dimensional subspace in $\left(\operatorname{ad}_{r} \mathcal{M}^{+}\right) \overline{\tau(2 \mu)}$ annihilated by all $\left(\operatorname{ad}_{r} x_{i}\right)$ for $\alpha_{i} \in \pi_{\Theta}$. Hence up to multiplication by a nonzero scalar, any highest weight vector $Y_{\lambda}^{\prime}$ of weight $\lambda$ in $\left(\operatorname{ad}_{r} U\right) \overline{\tau(2 \mu)}$ satisfies

$$
Y_{\lambda}^{\prime} \in Y \otimes \overline{\tau(2 \mu)} \otimes X+\sum_{0 \leq \eta<\mu-w_{0} \mu} K(2 \mu)_{\eta}^{-} \otimes \overline{\tau(2 \mu)} \otimes K(2 \mu)^{+} .
$$

Therefore, any two highest weight vectors of weight $\lambda$ in $\left(\operatorname{ad}_{r} U\right) \overline{\tau(2 \mu)}$ are linearly dependent. Hence (23) is proved.

By (25) and the discussion preceding the lemma, the weight $-\beta$ of $\overline{\tau(2 \mu)} \otimes X$ satisfies $\beta=\mu-w_{0}^{\prime} \mu$. On the other hand, $\beta$ is defined to be the weight $\mu-w_{0} \mu-\lambda$. Thus

$$
\lambda=w_{0}^{\prime} \mu-w_{0} \mu
$$

is a necessary condition for (24) to hold.

Assume now that $\lambda=w_{0}^{\prime} \mu-w_{0} \mu$ holds. Recall the isomorphism of right $U$ modules $\left(\operatorname{ad}_{r} U\right) \tau(2 \mu) \cong V(\mu)^{*} \otimes V\left(-w_{0} \mu\right)^{*}$ noted in Section 1.6. To complete the proof, it suffices to show that the left $U$-module $V(\mu) \otimes V\left(-w_{0} \mu\right)$ contains a highest weight vector of weight $\lambda=w_{0}^{\prime} \mu-w_{0} \mu$.

Let $M$ denote the $\mathcal{M}$-submodule of $V(\mu)$ generated by the highest weight vector $v_{\mu} \in V(\mu)$. Similarly, let $M^{*}$ denote the $\mathcal{M}$-submodule of $V\left(-w_{0} \mu\right)$ generated by the highest weight vector $v_{-w_{0} \mu} \in V\left(-w_{0} \mu\right)$. As $\lambda=w_{0}^{\prime} \mu-w_{0} \mu$ and $\left(\lambda, \alpha_{i}\right)=0$ for all $\alpha_{i} \in \pi_{\Theta}$ the $\mathcal{M}$-modules $M$ and $M^{*}$ are dual to each other. Hence the subset $M \otimes M^{*}$ of $V(\mu) \otimes V\left(-w_{0} \mu\right)$ contains a nonzero $\mathcal{M}$-invariant element $v$ of weight 
$w_{0}^{\prime} \mu-w_{0} \mu=\lambda$. But as $\lambda+\alpha_{i} \nless \mu-w_{0} \mu$ for all $\alpha_{i} \notin \pi_{\Theta}$, the element $v$ is a highest weight vector for the action of $U$ on $V(\mu) \otimes V\left(-w_{0} \mu\right)$. This completes the proof that the condition $\lambda=w_{0}^{\prime} \mu-w_{0} \mu$ implies (24).

\section{The triangular DeCOMPosition}

The aim of this section is to extract information about elements in $B$ and $F_{r}(\check{U})$ with respect to the triangular decomposition $\check{U} \cong G^{-} \otimes U^{+} \otimes \check{U}^{0}$. These results will be used to express certain elements in $F_{r}(\check{U})$ as elements of $\check{B}$ up to terms of lower $\mathcal{F}^{\theta}$ filter degree.

4.1. Triangular decomposition of elements in $\check{B}$. Recall the partial ordering $\geq_{r}$ defined in Section 1.4. Set

$$
T_{\geq}=\operatorname{span}\left\{\tau(\delta) \mid \delta \in P(\pi) \text { and } \tilde{\delta} \geq_{r} 0\right\} .
$$

Note that $T_{\geq}$is a subalgebra of $\check{U}^{0}$ which contains $\mathcal{C}\left[t_{i} \mid \alpha_{i} \in \pi\right]$ and $T_{\Theta}^{\prime}$.

Lemma 4.1. The algebra $\check{B}$ is a subset of $G^{-} U^{+} T_{\geq}$.

Proof. The relations of $U$ guarantee that the subalgebra of $U$ generated by $G^{-}$, $U^{+}, T_{\Theta}^{\prime}$, and $\mathcal{C}\left[t_{i} \mid \alpha_{i} \in \pi\right]$ is a subset of $G^{-} U^{+} T_{\geq}$. Note that by definition (7) each $B_{i}$ lies in the subalgebra generated by $G^{-}, U^{+}, T_{\Theta}$ and $\mathcal{C}\left[t_{i} \mid \alpha_{i} \in \pi\right]$. Moreover, $\mathcal{M} \subseteq G^{-} U^{+} T_{\Theta}$. This completes the proof of the lemma.

4.2. Triangular decomposition of elements in $\left(\operatorname{ad}_{r} U\right) \tau(2 \mu)$. It follows from (9) that $\mathcal{F}_{m}\left(F_{r}(\check{U})\right)$ is finite dimensional for all $m \geq 0$. Unfortunately, a similar statement does not hold for the filtration $\mathcal{F}^{\theta}$. In order to prove the main results of this paper, it is necessary to restrict to finite-dimensional subspaces of $\mathcal{F}_{m}^{\theta}\left(F_{r}(\check{U})\right)$. This is done by intersecting with spaces $G(\leq \mu)$ defined as follows. For $\mu \in P(\pi)$ set

$$
G(\leq \mu):=U^{-} G^{+} \tau(\mu) \mathcal{C}\left[t_{1}^{-2}, \ldots, t_{n}^{-2}\right]=\sum_{\eta \in Q^{+}(\pi)} U^{-} G^{+} \tau(\mu-2 \eta) .
$$

Note that the sets $G(\leq \mu)$ for $\mu \in P(\pi)$ satisfy the following multiplicative property:

$$
G\left(\leq \mu_{1}\right) G\left(\leq \mu_{2}\right)=G\left(\leq\left(\mu_{1}+\mu_{2}\right)\right) \text { for all } \mu_{1}, \mu_{2} \in P(\pi) .
$$

Proposition 4.2. Fix $\mu \in P^{+}(\pi)$. Suppose that $v \in\left(a d_{r} U\right) \tau(2 \mu)$ and $w \in$ $G^{-} U^{+} T_{\geq}$satisfy

$$
v-w \in \sum_{\alpha \nsupseteq 2 \mu} G(\leq \alpha) .
$$

Then $v \in G^{-} U^{+} T_{\geq}$.

Proof. The description of the right adjoint action (11) implies that $\left(\operatorname{ad}_{r} U\right) \tau(2 \mu)$ is a subset of $G(\leq 2 \mu)$. In particular, there exists $X \in U^{-} G^{+} \tau(2 \mu)$ such that

$$
v \in X+\sum_{\eta>0} U^{-} G^{+} \tau(2 \mu-2 \eta) .
$$

It now follows from (29) that

$$
w \in X+\sum_{\alpha \neq 2 \mu} U^{-} G^{+} \tau(\alpha) .
$$


On the other hand, since $w \in G^{-} U^{+} T_{\geq}$, we have

$$
w \in \sum_{\xi, \zeta} \sum_{\tilde{\gamma} \geq r 0} G_{-\zeta}^{-} U_{\xi}^{+} \tau(\gamma)=\sum_{\zeta, \xi} \sum_{\tilde{\gamma}-\tilde{\zeta}-\tilde{\xi} \geq_{r} 0} U_{-\zeta}^{-} G_{\xi}^{+} \tau(\gamma)
$$

where $\zeta$ and $\xi$ run over elements in $Q^{+}(\pi)$ and each $\gamma$ is in $P(\pi)$. It follows that

$$
X \in \sum_{\substack{\zeta, \xi \\ 2 \tilde{\mu} \geq r \tilde{\zeta}+\tilde{\xi}}} U_{-\zeta}^{-} G_{\xi}^{+} \tau(2 \mu) .
$$

By (31) and Lemma 2.2, there exists

$$
a \in \sum_{\substack{\zeta, \xi \\ 2 \tilde{\mu} \geq_{r} \tilde{\zeta}+\tilde{\xi}}} U_{-\zeta}^{-} U_{\xi}^{+}
$$

such that $v=\left(\operatorname{ad}_{r} a\right) \tau(2 \mu)$. Now (10) implies that

$$
\begin{aligned}
v & \in \sum_{\alpha, \beta} \sum_{2 \tilde{\eta} \leq r} \sum_{\alpha \tilde{\mu}-\tilde{\alpha}-\tilde{\beta}} U_{-\alpha}^{-} G_{\beta}^{+} \tau(2 \mu-2 \eta) \\
& =\sum_{\alpha, \beta} \sum_{2 \tilde{\eta} \leq_{r} 2 \tilde{\mu}-\tilde{\alpha}-\tilde{\beta}} G_{-\alpha}^{-} U_{\beta}^{+} \tau(2 \mu-2 \eta-\alpha-\beta) \\
& \subseteq \sum_{\alpha, \beta} \sum_{\left\{\delta \in P(\pi) \mid \tilde{\delta} \geq_{r} 0\right\}} G_{-\alpha}^{-} U_{\beta}^{+} \tau(\delta) .
\end{aligned}
$$

The proposition follows from the definition of $T_{\geq}$.

4.3. Triangular decomposition of elements in $\mathcal{F}_{m}^{\theta}(\check{U})$. Suppose that $a \in$ $\mathcal{F}_{m}^{\theta}\left(F_{r}(\check{U})\right)$ for some $m \in \frac{1}{N} \mathbb{Z}$. It follows from Proposition 2.4 that

$$
a \in \sum_{\left\{\gamma \in P^{+}(\pi) \mid h^{\Sigma}(2 \gamma) \leq m\right\}}\left(\operatorname{ad}_{r} U\right) \tau(2 \gamma) .
$$

Now suppose that $\mu \in P^{+}(\pi)$ such that $h^{\Sigma}(2 \mu)>m$. It further follows that $a \in \sum_{\alpha \nsupseteq 2 \mu} G(\leq \alpha)$. The next result can be viewed as an extension of Proposition 4.2 with $\left(\operatorname{ad}_{r} U\right) \tau(2 \mu)$ replaced by $F_{r}(\check{U})$.

Corollary 4.3. Suppose that $w \in G^{-} U^{+} T_{\geq}$and

$$
v \in \sum_{\left\{\gamma \in P^{+}(\pi) \mid h^{\Sigma}(2 \gamma)>m\right\}}\left(\operatorname{ad}_{r} U\right) \tau(2 \gamma)
$$

such that $v-w \in \mathcal{F}_{m}^{\theta}(\check{U})$ for some $m \in \frac{1}{N} \mathbb{Z}$. Then $v \in G^{-} U^{+} T_{\geq}$.

Proof. We can find distinct weights $\mu_{i} \in P^{+}(\pi)$, for $i=1, \ldots, r$, and elements $v_{i} \in\left(\operatorname{ad}_{r} U\right) \tau\left(2 \mu_{i}\right)$ such that $v=v_{1}+\cdots+v_{r}$. Furthermore, we may assume that $h^{\Sigma}\left(2 \mu_{i}\right) \geq m+\frac{1}{N}$ for each $1 \leq i \leq r$. Reorder the $\mu_{i}$ if necessary so that $\mu_{i}>\mu_{j}$ implies $i>j$. It follows that $v_{i} \in \sum_{\alpha \nsucceq 2 \mu_{r}} G(\leq \alpha)$ for $i=1, \ldots, r-1$. Moreover, $\mathcal{F}_{m}^{\theta}(\check{U})$ is a subset of $\sum_{\alpha \nsupseteq 2 \mu_{r}} G(\leq \alpha)$ and hence $v_{r}-w \in \sum_{\alpha \nsupseteq 2 \mu_{r}} G(\leq \alpha)$. An application of the preceding proposition yields $v_{r} \in G^{-} U^{+} T_{\geq}$. The corollary now follows by induction on $r$ after replacing $w$ by $w-v_{r}$ and $v$ by $v-v_{r}$. 


\section{The quantum IWASAWA DeCOMPOSition}

There is a second tensor product decomposition of $\check{U}$ involving the subalgebra $\check{B}$ called the quantum Iwasawa decomposition. Since we are interested in elements of $\check{B}$, it is often better to express elements in terms of the quantum Iwasawa decomposition. This section provides ways to translate information between the Iwasawa and the triangular decompositions.

5.1. The quantum Iwasawa decomposition of $\check{U}$. In general, the set $\widetilde{P(\pi)}=$ $\{\tilde{\alpha} \mid \alpha \in P(\pi)\}$ is not a subset of $P(\pi)$. As in Section 1.2, we enlarge $\check{T}$ to a group $\check{T}_{\Theta} \check{\mathcal{A}}$ such that the isomorphism $\tau$ extends to an isomorphism from $P(\pi)+\widetilde{P(\pi)}$ to $\check{T}_{\Theta} \check{\mathcal{A}}$. Set $\check{\mathcal{A}}=\{\tau(\tilde{\alpha}) \mid \alpha \in P(\pi)\}$ and $\check{T}_{\Theta}=\{\tau((\alpha+\Theta(\alpha)) / 2) \mid \alpha \in P(\pi)\}$. Note that $\check{T}_{\Theta} \cap \check{T}=T_{\Theta}^{\prime}$ and that $\check{T}_{\Theta} \check{\mathcal{A}}$ is isomorphic to the group $\check{T}_{\Theta} \times \check{\mathcal{A}}$. As in Section 1.2, we can form the algebra $\check{U} \check{T}_{\Theta}$ by insisting that relation (3) holds for all $\tau(\lambda) \in \check{T}_{\Theta}$. Note that it is possible that $\left(\tilde{\omega}_{i}, \alpha_{j}\right) \in(\mathbb{Z} / 2) \backslash \mathbb{Z}$. Hence, strictly speaking, the algebra $\check{U} \check{T}_{\Theta}$ is defined over the field $\mathcal{C}=\mathbb{C}\left(q^{1 / 2}\right)$.

Let ad denote the left adjoint action Let02, (1.2) and Section 5]. Set $N^{+}$equal to the subalgebra of $U^{+}$generated by the $\left(\operatorname{ad} \mathcal{M}^{+}\right)$-module $\left(\operatorname{ad} \mathcal{M}^{+}\right)\left(\mathcal{C}\left[x_{i} \mid \alpha_{i} \notin\right.\right.$ $\left.\left.\pi_{\Theta}\right]\right)$. The following isomorphism is a form of the quantum Iwasawa decomposition and easily deduced from [Let08, Section 4]. The multiplication map induces an isomorphism

$$
B \check{T}_{\Theta} \otimes \mathcal{C}[\check{\mathcal{A}}] \otimes N^{+} \cong \check{U} \check{T}_{\Theta}
$$

This implies in particular that $\check{U}=\left(B N^{+} \check{T}_{\Theta} \check{\mathcal{A}}\right) \cap \check{U}=B N^{+} \check{U}^{0}$.

The next lemma provides more detailed information concerning the triangular decomposition of elements $B_{I}$, for $I \in \mathcal{I}$.

Lemma 5.1. For all $I \in \mathcal{I}$, we have

$$
B_{I} \in(y t)_{I}+\sum_{\substack{\eta<\mathrm{wt}(I) \\ h^{\Sigma}(\eta)<h^{\Sigma}(\operatorname{wt}(I))}} G_{\eta}^{-} U^{+} T .
$$

Proof. This is a straightforward induction on $h(\mathrm{wt}(I))$.

5.2. Comparing the two tensor product decompositions. Estimates on the filter degree or weight of an element $u \in \check{U}$ are usually obtained by decomposing $u$ with respect to the triangular decomposition (2). A consequence of the following lemma is that the summands of the decomposition of $u$ with respect to the quantum Iwasawa decomposition often satisfy the same estimates.

Proposition 5.2. Assume $\lambda \in P(\pi)$ and $k \in \frac{1}{N} \mathbb{Z}$. Then the following relations hold:

(i) $G(\leq \lambda)=\sum_{I \in \mathcal{I}} B_{I}\left[G^{+} \check{U}^{0} \cap G(\leq(\lambda-\operatorname{wt}(I)))\right]$.

(ii) $\mathcal{F}_{k}^{\theta}(\check{U})=\sum_{I \in \mathcal{I}} B_{I}\left[G^{+} \check{U}^{0} \cap \mathcal{F}_{k-h^{\Sigma}(\operatorname{wt}(I))}^{\theta}(\check{U})\right]$.

(iii) $\sum_{h^{\Sigma}(\eta)<k} \check{U}_{-\eta}=\sum_{I \in \mathcal{I}} B_{I}\left[G^{+} \check{U}^{0} \cap \sum_{h^{\Sigma}(\eta+w t(I))<k} \check{U}_{-\eta}\right]$.

Proof. Note that for all three statements it suffices to show that the left hand side is contained in the right hand side. We first prove (i). Let $a \in G(\leq \lambda)$. Without loss of generality, we can assume that $a=(y t)_{I} a_{I}$ for some $I \in \mathcal{I}$ and $a_{I} \in U^{+} \check{T}$. We have to show that $(y t)_{I} a_{I}$ is also contained in the set on the right hand side 
of (i). Note that $a \in G(\leq \lambda)$ implies $a_{I} \in G(\leq(\lambda-\mathrm{wt}(I)))$. The multiplicative property (28) implies $B_{I} a_{I} \in G(\leq \lambda)$. By Lemma 5.1 one gets

$$
(y t)_{I} a_{I}-B_{I} a_{I} \in \sum_{\eta<\operatorname{wt}(I)} G_{-\eta}^{-} U^{+} T \cap G(\leq \lambda) .
$$

Claim (i) now follows by induction on the height of wt $(I)$.

One obtains assertions (ii) and (iii) using a similar argument with $G(\leq \lambda)$ replaced by $\mathcal{F}_{k}^{\theta}(\check{U})$ and $\sum_{h^{\Sigma}(\eta)<k} \check{U}_{-\eta}$, respectively.

\section{A projection of $\check{U}$ onto $\check{B}$}

This section defines a projection $b$ of $\check{U}$ onto $\check{B}$ using the quantum Iwasawa decomposition. We further show that $b(u)$ inherits estimates of filter degree and weight from $u$. The map $b$ is a critical tool in the construction of a particularly nice basis of the center of $\check{B}$ in Section 8

6.1. An $\left(\operatorname{ad}_{r} \mathcal{M} T_{\Theta}\right)$-submodule of $\check{U} \check{T}_{\Theta}$. Let $N_{+}^{+}$denote the intersection of $N^{+}$ with the augmentation ideal of $U$. Set $\check{\mathcal{A}}^{+}:=\check{\mathcal{A}} \backslash\{1\}$. Let $\mathcal{G}$ denote the subset $\check{\mathcal{A}} N_{+}^{+}+\check{\mathcal{A}}^{+}$of $\check{U} \check{T}_{\Theta}$.

Lemma 6.1. The vector space $B \check{T}_{\Theta} \mathcal{G}$ is an $\left(\operatorname{ad}_{r} \mathcal{M} T_{\Theta}\right)$-module. Moreover, $B \check{T}_{\Theta} \mathcal{G} \cap$ $B \check{T}_{\Theta}=\{0\}$.

Proof. The second statement follows immediately from the quantum Iwasawa decomposition (32).

To prove the first statement note first that $B \check{T}_{\Theta} \mathcal{G}$ is $\left(\operatorname{ad}_{r} T_{\Theta}\right)$-invariant. Moreover, $\left(\operatorname{ad}_{r} m\right) t^{\prime} \in \mathcal{M} t^{\prime}$ for all $m \in \mathcal{M}, t^{\prime} \in \check{\mathcal{A}}^{+}$and $\check{\mathcal{A}}^{+} \mathcal{M}=\mathcal{M} \check{\mathcal{A}}^{+}$. Thus it remains to prove that

$$
\left(\operatorname{ad}_{r} x_{i}\right) N_{+}^{+} \subseteq \mathcal{M} N_{+}^{+}, \quad\left(\operatorname{ad}_{r} y_{i}\right) N_{+}^{+} \subseteq \mathcal{M} N_{+}^{+}
$$

for all $\alpha_{i} \in \pi_{\Theta}$. In view of (11) these relations are equivalent to

$$
N_{+}^{+} x_{i} \subset \mathcal{M} N_{+}^{+}, \quad N_{+}^{+} y_{i} \subset \mathcal{M} N_{+}^{+}
$$

for all $\alpha_{i} \in \pi_{\Theta}$. The first formula follows immediately from the fact that the multiplication map gives an isomorphism $U^{+} \cong \mathcal{M}^{+} \otimes N^{+}$of vector spaces [Kéb99. For the second formula one has to commute $y_{i}$ to the left side using the defining relations (iii) of $U$ before applying $U^{+} \cong \mathcal{M}^{+} \otimes N^{+}$.

6.2. A $\mathcal{M} T_{\Theta}$-module decomposition. Recall that $B \check{T}_{\Theta}$ contains the Hopf algebra $\mathcal{M} T_{\Theta}$. It follows that $B \breve{T}_{\Theta}$ is an $\left(\operatorname{ad}_{r} \mathcal{M} T_{\Theta}\right)$-module. Thus the quantum Iwasawa decomposition (32) and Lemma 6.1 yield the following direct sum decomposition

$$
\check{U} \check{T}_{\Theta}=B \check{T}_{\Theta} \mathcal{G} \oplus B \check{T}_{\Theta}
$$

of $\check{U} \check{T}_{\Theta}$ into $\mathcal{M} T_{\Theta}$-modules with respect to the right adjoint action. Given $u \in \check{U} \check{T}_{\Theta}$, let $b(u)$ be the projection of $u$ onto $\check{B} \check{T}_{\Theta}$ with respect to (33).

Lemma 6.2. The projection $b$ restricts to a mapping of $\check{U}$ onto $\check{B}$. 
Proof. The quantum Iwasawa decomposition for $\check{U}$ below (32) implies that $b(\check{U})=$ $b\left(B \check{U}^{0}\right)$. Any element $u \in B \check{U}^{0}$ can be uniquely decomposed in the form $u=u_{1}+u_{2}$ where

$$
u_{1} \in \sum_{\Theta(\lambda) \neq \lambda} B \tau(\lambda), \quad u_{2} \in \sum_{\Theta(\lambda)=\lambda} B \tau(\lambda) .
$$

The claim of the lemma follows because $b(u)=u_{2}$ and $u_{2} \in \check{B}$.

6.3. Properties of the map $b$. Consider $u \in \check{U}$. The next result shows that $b(u)$ retains certain characteristics of $u$ related to degree and weight.

Proposition 6.3. Assume $\lambda \in P(\pi)$ and $k \in \frac{1}{N} \mathbb{Z}$. The map $b$ has the following properties.

(i) $b(G(\leq \lambda)) \subseteq G(\leq \lambda)$.

(ii) $b\left(\mathcal{F}_{k}^{\theta}(\check{U})\right) \subseteq \mathcal{F}_{k}^{\theta}(\check{U})$.

(iii) $b\left(\sum_{h^{\Sigma}(\eta)<k} \check{U}_{-\eta}\right) \subseteq \sum_{h^{\Sigma}(\eta)<k} \check{U}_{-\eta}$.

Proof. We prove assertion (i) using Proposition 5.2(i). The other two assertions follow in a similar manner from Proposition [5.2(ii) and (iii). Assume $u \in G(\leq \lambda)$. We can write

$$
u=\sum_{I \in \mathcal{I}} \sum_{\gamma \in Q^{+}(\pi)} \sum_{\beta \in P(\pi)} B_{I} a_{I \gamma \beta}
$$

where $a_{I \gamma \beta} \in G_{\gamma}^{+} \tau(\beta)$ and the set $\left\{a_{I \gamma \beta} \mid \gamma \in Q^{+}(\pi)\right.$ and $\left.\beta \in P(\pi)\right\}$ is linearly independent for fixed $I \in \mathcal{I}$. By Proposition $5.2(\mathrm{i})$ one gets

$$
\sum_{\gamma \in Q^{+}(\pi)} \sum_{\beta \in P(\pi)} a_{I, \gamma, \beta} \in G(\leq(\lambda-\mathrm{wt}(I)))
$$

for each $I \in \mathcal{I}$. Hence $a_{I, \gamma, \beta} \in G(\leq(\lambda-\operatorname{wt}(I)))$ for all $I \in \mathcal{I}, \gamma \in Q^{+}(\pi)$, and $\beta \in P(\pi)$. The multiplicative property (28) and $B_{i} \in G\left(\leq \alpha_{i}\right)$ ensure that $B_{I} \in G(\leq \mathrm{wt}(I))$. Hence $B_{I} a_{I \gamma \beta} \in G(\leq \lambda)$ for all $I, \gamma, \beta$. On the other hand, the definition of $b$ implies that

$$
b(u)=\sum_{I \in \mathcal{I}} \sum_{\tilde{\gamma}=0} \sum_{\tilde{\beta}=0} B_{I} a_{I \gamma \beta} .
$$

Therefore, $b(u) \in G(\leq \lambda)$.

\section{Highest weight vectors in $G^{-} \mathcal{M}^{+} T_{\Theta}^{\prime}$}

Let $\mathcal{V}$ be the vector space defined by

$$
\mathcal{V}=G^{-} \mathcal{M}^{+} T_{\Theta}^{\prime} \cap F_{r}(\check{U})^{U^{-}} \cap \sum_{\lambda \in 2 P^{+}(\Sigma)} \check{U}_{-\lambda} .
$$

In other words, $\mathcal{V}$ is the span of all highest weight vectors of spherical weight contained in $G^{-} \mathcal{M}^{+} T_{\Theta}^{\prime} \cap F_{r}(\check{U})$.

The main result of this section finds a basis of $\mathcal{V}$. This basis will be used in Section 8 to find a basis of $Z(\check{B})$. Both bases are indexed by the subset $P_{Z(\check{B})}$ of $P^{+}(\pi)$ defined by (34) below. Moreover, in Section 7.3 we obtain information about the weight spaces of spherical submodules of $F_{r}(\check{U})$ generated by highest weight vectors not contained in $G^{-} \mathcal{M}^{+} T_{\Theta}^{\prime}$. 
7.1. Highest weight vectors for spherical modules. The next proposition determines which values $\mu \in P^{+}(\pi)$ satisfy the following condition: $\left(\operatorname{ad}_{r} U\right) \tau(2 \mu)$ contains a highest weight vector of spherical weight that is also an element of $G^{-} \mathcal{M}^{+} T_{\Theta}^{\prime}$. Recall that $w_{0}$ is the longest element of the Weyl group $W$ and that $w_{0}^{\prime}$ is the longest element of the subgroup $W\left(\pi_{\Theta}\right)$ of $W$ (see Section 3.2). Let $P_{Z(\check{B})}$ be the subset of $P^{+}(\pi)$ defined by

$$
P_{Z(\check{B})}=\left\{\mu \in P^{+}(\pi) \mid \Theta(\mu)=\mu+w_{0} \mu-w_{0}^{\prime} \mu\right\} .
$$

Proposition 7.1. Let $\mu \in P^{+}(\pi)$. Then $\left(\operatorname{ad}_{r} U\right) \tau(2 \mu) \cap \mathcal{V} \neq\{0\}$ if and only if $\mu \in P_{Z(\check{B})}$. Moreover, in this case any nonzero element $Y_{\lambda} \in\left(\operatorname{ad}_{r} U\right) \tau(2 \mu) \cap \mathcal{V}$ is a weight vector of spherical weight $\lambda=2 \tilde{\mu}$, and $\operatorname{dim}\left(\left(\operatorname{ad}_{r} U\right) \tau(2 \mu) \cap \mathcal{V}\right)=1$.

Proof. Fix $\mu \in P^{+}(\pi)$. Assume first that $\mu \in P_{Z(\check{B})}$ and set $\lambda=2 \tilde{\mu}$. By Let08, Section 2], $\left\{\tilde{\gamma} \mid \gamma \in P^{+}(\pi)\right\}$ is a subset of $P^{+}(\Sigma)$. Hence $\lambda$ is a spherical weight. Note that $2 \tilde{\mu}=\mu-\Theta(\mu)$. Thus, the definition of $P_{Z(\check{B})}$ ensures that $\lambda=w_{0}^{\prime} \mu-w_{0} \mu$. Hence

$$
\mu-w_{0} \mu-\lambda=\mu-w_{0}^{\prime} \mu \in \mathbb{N} \pi_{\Theta} .
$$

By Theorem 3.2 there exists a highest weight vector $Y_{\lambda} \in\left(\operatorname{ad}_{r} U\right) \tau(2 \mu)$ of weight $\lambda$ with respect to the right adjoint action. Moreover, $Y_{\lambda}$ is unique up to nonzero scalar multiplication. Thus the assertion $\operatorname{dim}\left(\left(\operatorname{ad}_{r} U\right) \tau(2 \mu) \cap \mathcal{V}\right)=1$ follows once we establish below that $Y_{\lambda} \in G^{-} \mathcal{M}^{+} T_{\Theta}^{\prime}$.

In the graded algebra $\mathrm{Gr}$ the corresponding highest weight vector

$$
\bar{Y}_{\lambda} \in\left(\operatorname{ad}_{r} U\right) \overline{\tau(2 \mu)}
$$

of weight $\lambda$ satisfies

$$
\bar{Y}_{\lambda} \in \sum_{\alpha \leq \mu-w_{0} \mu} K(2 \mu)_{\alpha}^{-} \otimes \mathcal{C} \overline{\tau(2 \mu)} \otimes K(2 \mu)_{\lambda-\alpha}^{+} .
$$

By Lemma 2.2 and the fact that $Y_{\lambda}$ has weight $\lambda$ with respect to the right adjoint action, there exists

$$
a \in \sum_{\zeta \leq \mu-w_{0} \mu} U_{-\zeta}^{-} U_{\zeta-\lambda}^{+}
$$

such that $\left(\operatorname{ad}_{r} a\right) \overline{\tau(2 \mu)}=\bar{Y}_{\lambda}$. Inclusion (10) ensures that

$$
Y_{\lambda} \in \sum_{\zeta \leq \mu-w_{0} \mu} \sum_{\alpha \leq \zeta} \sum_{0 \leq \eta \leq \zeta-\lambda} G_{-\alpha}^{-} U_{\alpha-\lambda}^{+} \tau(2 \mu-\lambda-2 \eta) .
$$

Note that the inequalities of weights under the summations imply $\alpha-\lambda \in \mathbb{N} \pi_{\Theta}$ and $\eta \in \mathbb{N} \pi_{\Theta}$. Moreover, by definition $\lambda=\mu-\Theta(\mu)$ and hence

$$
\tau(2 \mu-\lambda-2 \eta)=\tau(\mu+\Theta(\mu)-2 \eta) \in T_{\Theta}^{\prime} .
$$

Combining (36), (37), and $\alpha-\lambda \in \mathbb{N} \pi_{\Theta}$ one obtains $Y_{\lambda} \in G^{-} \mathcal{M}^{+} T_{\Theta}^{\prime}$ and hence $Y_{\lambda} \in \mathcal{V} \cap\left(\operatorname{ad}_{r} U\right) \tau(2 \mu)$.

Now assume that $\left(\operatorname{ad}_{r} U\right) \tau(2 \mu)$ contains a highest weight spherical vector $Y_{\lambda}$ of weight $\lambda$ with respect to the right adjoint action. Assume further that $Y_{\lambda} \in$ $G^{-} \mathcal{M}^{+} T_{\Theta}^{\prime}$. In view of the isomorphism $g:\left(\operatorname{ad}_{r} U\right) \tau(2 \mu) \rightarrow\left(\operatorname{ad}_{r} U\right) \overline{\tau(2 \mu)}$ given by 
(17), Lemma 3.1 implies that there exists a nonzero $Z \in U_{-\mu+w_{0} \mu}^{-} G^{+} \tau(2 \mu)$ such that

$$
Y_{\lambda} \in Z+\sum_{\alpha \geq 0} \sum_{\gamma<\mu-w_{0} \mu} U_{-\gamma}^{-} G^{+} \tau(2 \mu-\alpha) .
$$

Since $Y_{\lambda}$ has weight $\lambda$, it follows that $Z$ is a weight vector of weight $\lambda$. Hence

$$
Z \in G_{-\mu+w_{0} \mu}^{-} U_{\mu-w_{0} \mu-\lambda}^{+} \tau(2 \mu-\lambda) \text {. }
$$

The fact that $Y \in G^{-} \mathcal{M}^{+} T_{\Theta}^{\prime}$ ensures that $Z$ is in $G_{-\mu+w_{0} \mu}^{-} \mathcal{M}^{+} T_{\Theta}^{\prime}$. Thus $\mu-w_{0} \mu-$ $\lambda \in \mathbb{N} \pi_{\Theta}$ and $\Theta(2 \mu-\lambda)=2 \mu-\lambda$. Since $\lambda$ is spherical, we have that $\Theta(\lambda)=-\lambda$. Hence $\lambda=\mu-\Theta(\mu)=2 \tilde{\mu}$. Theorem 3.2 further implies that $\lambda=w_{0}^{\prime} \mu-w_{0} \mu$. Thus $\mu-\Theta(\mu)=w_{0}^{\prime} \mu-w_{0} \mu$ and so $\mu \in P_{Z(\check{B})}$.

7.2. Highest weight spherical vectors in $G^{-} \mathcal{M}^{+} T_{\Theta}^{\prime}$. Fix $\mu \in P_{Z(\check{B})}$. By Proposition 7.1, there exists a unique (up to nonzero scalar multiplication) highest weight vector of weight $2 \tilde{\mu}$ in $G^{-} \mathcal{M}^{+} T_{\Theta}^{\prime} \cap\left(\operatorname{ad}_{r} U\right) \tau(2 \mu)$ with respect to the right adjoint action. Denote this highest weight vector by $Y^{\mu}$. Note that $\mu=0$ is in $P_{Z(\check{B})}$ and $Y^{0}$ is just a nonzero scalar. Moreover, $Y^{\mu} \in \mathcal{V}$ for all $\mu \in P_{Z(\check{B})}$.

Theorem 7.2. The set of $\left\{Y^{\mu} \mid \mu \in P_{Z(\check{B})}\right\}$ is basis for $\mathcal{V}$.

Proof. Note that each $Y^{\mu} \in\left(\operatorname{ad}_{r} U\right) \tau(2 \mu) \backslash\{0\}$. Hence the set $\left\{Y^{\mu} \mid \mu \in P_{Z(\check{B})}\right\}$ is linearly independent.

Let $Y \in \mathcal{V}$. Note that $\mathcal{V}$ can be written as a direct sum of its weight spaces. Hence we may reduce to the case where $Y$ is a weight vector of weight $\lambda$. Moreover, the definition of $\mathcal{V}$ ensures that $\lambda$ is a spherical weight. Since $Y \in F_{r}(\check{U})$, we can write $Y=\sum_{\gamma \in P^{+}(\pi)} a_{\gamma}$ with $a_{\gamma} \in\left(\operatorname{ad}_{r} U\right) \tau(2 \gamma)$. It follows from (10) that

$$
a_{\gamma} \in \sum_{\eta, \xi \geq 0} U_{-\lambda-\xi}^{-} G_{\xi}^{+} \tau(2 \gamma-2 \eta) \subseteq \sum_{\eta \geq 0} G^{-} U^{+} \tau(2 \gamma-\lambda-2 \eta) .
$$

By Proposition 7.1 it suffices to show that

$$
a_{\gamma} \in G^{-} \mathcal{M}^{+} T_{\Theta}^{\prime}
$$

for all $\gamma$. We prove relation (39) by induction on $m=\max \left\{h(\gamma) \mid a_{\gamma} \neq 0\right.$ ) $\}$. If $m=0$, then $Y=a_{0}$ and (39) holds. Assume $m>0$ and let $\bar{Y}=\sum_{h(\gamma)=m} \bar{a}_{\gamma}$ denote the element represented by $Y$ in $\mathrm{Gr}_{2 m}$. The relation $Y \in G^{-} \mathcal{M}^{+} T_{\Theta}^{\prime}$ implies

$$
\bar{a}_{\gamma} \in \sum_{\alpha \in \mathbb{N} \pi_{\ominus}} K(2 \gamma)^{-} \otimes \overline{\tau(2 \gamma)} \otimes K(2 \gamma)_{\alpha}^{+}
$$

for all $\gamma$ such that $h(\gamma)=m$. By Lemma 2.2 using $a_{\gamma} \in \check{U}_{-\lambda}$ one obtains

$$
a_{\gamma} \in \sum_{\xi \in \mathbb{N} \pi_{\Theta}}\left(\operatorname{ad}_{r} U_{-\lambda-\xi}^{-} U_{\xi}^{+}\right) \tau(2 \gamma) .
$$

Hence by (10) for all $\gamma$ with $h(\gamma)=m$ one obtains the following refinement of (38)

$$
a_{\gamma} \in \sum_{\eta, \xi \in \mathbb{N} \pi_{\Theta}} U_{-\lambda-\xi}^{-} G_{\xi}^{+} \tau(2 \gamma-2 \eta) \subseteq \sum_{\eta \in \mathbb{N} \pi_{\Theta}} G^{-} \mathcal{M}^{+} \tau(2 \gamma-\lambda-2 \eta) .
$$

For all $\gamma$ with $h(\gamma)=m$, as $\bar{a}_{\gamma} \neq 0$, there exists a nonzero

$$
X_{\gamma} \in \sum_{\xi \in \mathbb{N} \pi_{\ominus}} U_{-\lambda-\xi}^{-} G_{\xi}^{+} \tau(2 \gamma) \subseteq G^{-} \mathcal{M}^{+} \tau(2 \gamma-\lambda)
$$


such that

$$
a_{\gamma} \in X_{\gamma}+\sum_{\eta<\gamma} \sum_{\xi \in \mathbb{N} \pi_{\Theta}} U_{-\lambda-\xi}^{-} G_{\xi}^{+} \tau(2 \eta) \subseteq X_{\gamma}+\sum_{\eta<\gamma} G^{-} \mathcal{M}^{+} \tau(2 \eta-\lambda) .
$$

The maximality of $\gamma$ and the relation $Y \in G^{-} \mathcal{M}^{+} T_{\Theta}^{\prime}$ now imply that $\tau(2 \gamma-\lambda) \in T_{\Theta}^{\prime}$ for all $\gamma$ such that $h(\gamma)=m$. Together with (40) this yields $a_{\gamma} \in G^{-} \mathcal{M}^{+} T_{\Theta}^{\prime}$ for all $\gamma$ such that $h(\gamma)=m$. Hence

$$
\sum_{h(\gamma)<m} a_{\gamma}=Y-\sum_{h(\gamma)=m} a_{\gamma} \in G^{-} \mathcal{M}^{+} T_{\Theta}^{\prime}
$$

and by the inductive hypothesis $a_{\gamma} \in G^{-} \mathcal{M}^{+} T_{\Theta}^{\prime}$ for all $\gamma$.

7.3. $B$-invariant subspaces of $G(\leq 2 \mu)$. Fix $\mu \in P_{Z(\check{B})}$ and recall the definition of $Y^{\mu}$ given in Section 7.2. Recall (11) and let $v_{\mu}^{B}$ be the uniquely determined nonzero $\left(\operatorname{ad}_{r} B\right)$-invariant element of $\left(\operatorname{ad}_{r} U\right) Y^{\mu}$ such that

$$
v_{\mu}^{B} \in Y^{\mu}+\sum_{\{\gamma \in P(\Sigma) \mid} \check{U}_{-\gamma} .
$$

Recall further the definition of $G(\leq 2 \mu)$ given in (27). Set $V_{0}^{\mu}$ equal to the sum of all the spherical submodules of $G(\leq 2 \mu)$ of highest weight unequal to $2 \tilde{\mu}$.

Consider $\lambda \in P^{+}(\pi)$. As discussed at the beginning of Section 3.1, the highest weight of $\left(\operatorname{ad}_{r} U^{-}\right) \overline{\tau(2 \lambda)}$ with respect to the right adjoint action is $\lambda-w_{0} \lambda$. It follows from the description of $\left(\operatorname{ad}_{r} U\right) \overline{\tau(2 \lambda)}$ in (16) that the highest weight of $\left(\operatorname{ad}_{r} U\right) \overline{\tau(2 \lambda)}$ is $\lambda-w_{0} \lambda$. Using the isomorphism (17) we see that

$$
\left(\operatorname{ad}_{r} U\right) \tau(2 \lambda) \subseteq \sum_{\gamma \leq \lambda-w_{0} \lambda} \check{U}_{-\gamma} .
$$

Lemma 7.3. Let $\mu \in P_{Z(\check{B})}$. Then $\left(\left(\operatorname{ad}_{r} U\right) \tau(2 \eta)\right)^{B} \subseteq\left(V_{0}^{\mu}\right)^{B}$ for all $\eta \in P^{+}(\pi)$ satisfying $\eta<\mu$ and $\tilde{\eta}<\tilde{\mu}$. Moreover, $\left(V_{0}^{\mu}\right)^{B}=G(\leq 2 \mu)^{B} \cap \sum_{h^{\Sigma}(\gamma)<h^{\Sigma}(2 \mu)} \check{U}_{-\gamma}$.

Proof. The assumption $\eta<\mu$ forces $-w_{0} \eta<-w_{0} \mu$ and hence $\eta-w_{0} \eta<\mu-w_{0} \mu$. Since $\tilde{\eta}<_{r} \tilde{\mu}$ we further get $\tilde{\eta}-\widetilde{w_{0} \eta}<_{r} \tilde{\mu}-\widetilde{w_{0} \mu}$. Hence by (42) and (11), we see that

$$
\left(\left(\operatorname{ad}_{r} U\right) \tau(2 \eta)\right)^{B} \subseteq \sum_{\gamma<_{r} \tilde{\mu}-\widetilde{w_{0} \mu}} \check{U}_{-\gamma} .
$$

Note that $\widetilde{w_{0}^{\prime} \mu}=\tilde{\mu}$ and hence $-\widetilde{w_{0} \mu}=\tilde{\mu}$ for all $\mu \in P_{Z(\check{B})}$. Suppose that $\beta$ is a spherical weight such that $V(\beta)^{*}$ is isomorphic to a submodule of $\left(\operatorname{ad}_{r} U\right) \tau(2 \eta)$. By (43), we see that $\beta<_{r} \tilde{\mu}-\widehat{w_{0} \mu}=2 \tilde{\mu}$. Hence $\beta \neq 2 \tilde{\mu}$. Therefore, $\left(\left(\operatorname{ad}_{r} U\right) \tau(2 \eta)\right)^{B} \subseteq$ $\left(V_{0}^{\mu}\right)^{B}$ which proves the first assertion. To prove the second assertion suppose first that $V$ is a finite-dimensional simple spherical submodule of $G(\leq 2 \mu)$ contained in $\sum_{h^{\Sigma}(\gamma)<h^{\Sigma}(2 \mu)} \check{U}_{-\gamma}$. It follows that the highest weight of $V$ is unequal to $2 \tilde{\mu}$. Hence

$$
G(\leq 2 \mu)^{B} \cap\left(\sum_{h^{\Sigma}(\gamma)<h^{\Sigma}(2 \mu)} \check{U}_{-\gamma}\right) \subseteq\left(V_{0}^{\mu}\right)^{B} .
$$

It now remains to show that

$$
\left(V_{0}^{\mu}\right)^{B} \cap\left(\operatorname{ad}_{r} U\right) \tau(2 \eta) \subseteq \sum_{h^{\Sigma}(\gamma)<h^{\Sigma}(2 \mu)} \check{U}_{-\gamma}
$$


for all $\eta \leq \mu$. Suppose that $V(\beta)^{*}$ is isomorphic to a submodule of $\left(V_{0}^{\mu}\right)^{B}$ with $\beta$ a spherical weight. It follows that $\beta=\tilde{\beta}$. The highest weight space of $\left(\operatorname{ad}_{r} U\right) \tau(2 \eta)$ is of weight $\eta-w_{0} \eta \leq \mu-w_{0} \mu$. Hence $\beta \leq_{r} \tilde{\eta}-\widetilde{w_{0} \eta} \leq_{r} \tilde{\mu}-\widetilde{w_{0} \mu}=2 \tilde{\mu}$. The definition of $V_{\mu}^{0}$ forces $\beta \neq 2 \tilde{\mu}$ and thus $\beta<_{r} 2 \tilde{\mu}$.

\section{A BASIS FOR THE CENTER $Z(\check{B})$}

Fix $\mu \in P_{Z(\check{B})}$ and recall the definitions of $Y^{\mu}, v_{\mu}^{B}$, and $V_{0}^{\mu}$ from Sections 7.2 and 7.3. In this section we show how to use the spherical vector $v_{\mu}^{B}$ to construct an element $d_{\mu}$ in $Z(\check{B}) \cap G(\leq 2 \mu)$ with a nonzero component in $\left(\operatorname{ad}_{r} U\right) \tau(2 \mu)$. We then prove that the set $\left\{d_{\mu} \mid \mu \in P_{Z(\check{B})}\right\}$ is a basis for $Z(\check{B})$.

8.1. Formulas for the right adjoint action. Let $\alpha_{k} \in \pi \backslash \pi_{\Theta}$. By [Let02, (7.15)] we have the following formula for the coproduct of $B_{k}$ :

$$
\Delta\left(B_{k}\right) \in t_{k} \otimes B_{k}+U \otimes \mathcal{M} T_{\Theta} .
$$

Moreover, recall from (77) that $\varepsilon\left(B_{k}\right)=s_{k}$ for all $\alpha_{k} \in \pi \backslash \pi_{\Theta}$.

Lemma 8.1. If $G$ is an $\left(\operatorname{ad}_{r} \mathcal{M} T_{\Theta}\right)$-invariant element of $\check{U}$, then

$$
\left(\operatorname{ad}_{r} B_{k}\right) G-s_{k} G=t_{k}^{-1}\left(G B_{k}-B_{k} G\right)
$$

for all $\alpha_{k} \in \pi \backslash \pi_{\Theta}$.

Proof. Fix $\alpha_{k} \in \pi \backslash \pi_{\Theta}$. By (44) we have $\Delta\left(B_{k}\right)=t_{k} \otimes B_{k}+\sum_{i} a_{i} \otimes b_{i}$ where $a_{i} \in U$ and $b_{i} \in \mathcal{M} T_{\Theta}$. Hence $s_{k}=\epsilon\left(B_{k}\right)=\sigma\left(t_{k}\right) B_{k}+\sum_{i} \sigma\left(a_{i}\right) b_{i}$ and therefore $\sum_{i} \sigma\left(a_{i}\right) b_{i}=-t_{k}^{-1} B_{k}+s_{k}$. Let $G$ be an $\left(\operatorname{ad}_{r} \mathcal{M} T_{\Theta}\right)$-invariant element of $\check{U}$. Since $\mathcal{M} T_{\Theta}$ is a Hopf subalgebra of $\check{U}$, it follows that $G$ commutes with elements of $\mathcal{M} T_{\Theta}$. Hence

$$
\begin{aligned}
\left(\operatorname{ad}_{r} B_{k}\right) G & =t_{k}^{-1} G B_{k}+\sum_{i} \sigma\left(a_{i}\right) G b_{i}=t_{k}^{-1} G B_{k}+\sum_{i} \sigma\left(a_{i}\right) b_{i} G \\
& =t_{k}^{-1} G B_{k}-t_{k}^{-1} B_{k} G+s_{k} G .
\end{aligned}
$$

8.2. $B$-invariant elements of $G(\leq 2 \mu)$. The next technical lemma will be used to complete the inductive step in the proof of Theorem 8.3 .

Lemma 8.2. Let $\mu \in P_{Z(\check{B})}$ and $v \in v_{\mu}^{B}+\left(V_{0}^{\mu}\right)^{B}$. Assume that there exists $m \in \frac{1}{N} \mathbb{Z}$ such that

$$
v-b(v) \in \sum_{h^{\Sigma}(\eta)<m} \check{U}_{-\eta} .
$$

Then there exists $w \in v_{\mu}^{B}+\left(V_{0}^{\mu}\right)^{B}$ such that $w-b(w) \in \mathcal{F}_{m-\frac{1}{N}}^{\theta}(\check{U})$.

Proof. Without loss of generality, we may assume that

$$
v-b(v) \notin \mathcal{F}_{m-\frac{1}{N}}^{\theta}(\check{U})
$$

because otherwise $w=v$ fulfills the claim of the lemma.

Since $v \in \check{U}^{B}$, it follows that $v$ is $\left(\operatorname{ad}_{r} \mathcal{M} T_{\Theta}\right)$-invariant. Recall that $b$ is a projection of $\mathcal{M} T_{\Theta}$-modules. Hence $b(v)$ is also $\left(\operatorname{ad}_{r} \mathcal{M} T_{\Theta}\right)$-invariant. Fix $\alpha_{k} \in \pi \backslash \pi_{\Theta}$. By Lemma 8.1 we have

$$
\left(\operatorname{ad}_{r} B_{k}\right)(v-b(v))-s_{k}(v-b(v))=t_{k}^{-1}\left[(v-b(v)) B_{k}-B_{k}(v-b(v))\right] .
$$


Hence, (45) and the definition (77) of $B_{i}$ ensure that

$$
\left(\operatorname{ad}_{r} B_{k}\right)(v-b(v))-s_{k}(v-b(v)) \in \sum_{h^{\Sigma}(\eta)<m+1} \check{U}_{-\eta} .
$$

On the other hand, the fact that $v \in \check{U}^{B}$ and $b(v) \in \check{B}$ implies that

$$
\begin{aligned}
\left(\operatorname{ad}_{r} B_{k}\right)(v-b(v))-s_{k}(v-b(v)) & =-t_{k}^{-1}\left[b(v) B_{k}-B_{k} b(v)\right] \\
& \in t_{k}^{-1} \sum_{I \in \mathcal{I}} B_{I} \mathcal{M}^{+} T_{\Theta}^{\prime} .
\end{aligned}
$$

Let $r$ be the smallest integer such that

$$
\left(\operatorname{ad}_{r} B_{k}\right)(v-b(v))-s_{k}(v-b(v))=t_{k}^{-1} \sum_{h^{\Sigma}(\operatorname{wt}(I)) \leq r} B_{I} m_{I}
$$

where each $m_{I} \in \mathcal{M}^{+} T_{\Theta}^{\prime}$. Note that the assumption on $r$ forces at least one element of the set $\left\{m_{I} \mid h^{\Sigma}(\operatorname{wt}(I))=r\right\}$ to be nonzero. By Lemma 5.1, it follows that

$$
\begin{aligned}
\left(\operatorname{ad}_{r} B_{k}\right)(v & -b(v))-s_{k}(v-b(v)) \\
& \in \sum_{h^{\Sigma}(\operatorname{wt}(I))=r} t_{k}^{-1}\left[(y t)_{I} m_{I}+\sum_{\eta<\operatorname{wt}(I)} U_{-\eta}^{-} G^{+} \check{U}^{0}\right] .
\end{aligned}
$$

Examining the right hand side of (49), we see that the lowest weight term of $\left(\operatorname{ad}_{r} B_{k}\right)(v-b(v))-s_{k}(v-b(v))$ written as a sum of nonzero weight vectors is contained in $\sum_{h^{\Sigma}(\eta)=r} \breve{U}_{-\eta}$. Hence (47) implies that $r<m+1$. Note, moreover, that $B_{I} \in \mathcal{F}_{r}^{\theta}(\check{U})$ if $h^{\Sigma}(\operatorname{wt}(I)) \leq r$. It now follows directly from (48) that

$$
\left(\operatorname{ad}_{r} B_{k}\right)(v-b(v))-s_{k}(v-b(v)) \in \mathcal{F}_{m-\frac{1}{N}}^{\theta}(\check{U}) .
$$

Let $s$ denote the degree of $v-b(v)$ with respect to the filtration $\mathcal{F}^{\theta}$. By (46), we see that $s \geq m$. By definition of $B_{k}$ an $\left(\mathcal{M} T_{\Theta}\right)$-invariant element $w$ of a $B$-module is $B$-invariant if and only if $B_{k} w-s_{k} w=0$ for all $\alpha_{k} \in \pi \backslash \pi_{\Theta}$. Hence (50) implies that the image of $v-b(v)$ in $\mathcal{F}_{s}^{\theta}(\check{U}) / \mathcal{F}_{s-\frac{1}{N}}^{\theta}(\check{U})$ is $B$-invariant.

By assumption, $v \in G(\leq 2 \mu)$. Hence, by Proposition 6.3(i) one has $b(v) \in$ $G(\leq 2 \mu)$. It follows that $h^{\Sigma}(2 \mu) \geq s \geq m$. Theorem 2.5 and Proposition 2.4 ensure that there exists $v^{\prime} \in G(\leq 2 \mu)^{B}$ such that

$$
v-b(v) \in v^{\prime}+\mathcal{F}_{m-\frac{1}{N}}^{\theta}(\check{U}) .
$$

The relation $h^{\Sigma}(2 \mu) \geq m$, assumption (45), and Lemma 7.3 imply that $v^{\prime} \in\left(V_{0}^{\mu}\right)^{B}$.

Set $w=v-v^{\prime}$. Note that $w \in v+\left(V_{0}^{\mu}\right)^{B}=v_{\mu}^{B}+\left(V_{0}^{\mu}\right)^{B}$. By the definition of the projection map $b$, we have that $v-b(v) \in B \check{T}_{\Theta} \mathcal{G}$. Hence $v^{\prime} \in B \check{T}_{\Theta} \mathcal{G}+\mathcal{F}_{m-\frac{1}{N}}^{\theta}(\check{U})$. By Proposition [6.3(ii), we obtain $b\left(v^{\prime}\right) \in \mathcal{F}_{m-\frac{1}{N}}^{\theta}(\check{U})$. Therefore, $w-b(w)=$ $\left[v-b(v)-v^{\prime}\right]+b\left(v^{\prime}\right) \in \mathcal{F}_{m-\frac{1}{N}}^{\theta}(\check{U})$.

8.3. Finding central elements. Suppose that $\mu \in P_{Z(\check{B})}$. The next theorem shows how to construct an element of $Z(\check{B})$ that is also contained in $G(\leq 2 \mu)$.

Theorem 8.3. Given $\mu \in P_{Z(\check{B})}$, there exists an element $d_{\mu} \in Z(\check{B})$ which lies in $v_{\mu}^{B}+\left(V_{0}^{\mu}\right)^{B}$. Moreover,

$$
d_{\mu} \in Y^{\mu}+\sum_{h^{\Sigma}(\gamma)<h^{\Sigma}(2 \mu)} \check{U}_{-\gamma}
$$


Proof. Set $V=\left(V_{0}^{\mu}\right)^{B}$. The definition (41) of $v_{\mu}^{B}$ and Lemma 7.3 imply that $v_{\mu}^{B}+V \subseteq Y^{\mu}+\sum_{h^{\Sigma}(\gamma)<h^{\Sigma}(2 \mu)} \check{U}_{-\gamma}$. Hence (51) follows from the first assertion of the theorem.

Note that the first assertion of the theorem holds provided we prove $\left(v_{\mu}^{B}+V\right) \cap$ $\check{B} \neq \emptyset$. It suffices to show that there exists $v \in v_{\mu}^{B}+V$ such that $v=b(v)$. Assume on the contrary that $v-b(v) \neq 0$ for all $v \in v_{\mu}^{B}+V$. It follows that for each $v \in v_{\mu}^{B}+V$, there exists a smallest rational number $n(v)$ such that

$$
v-b(v) \in \mathcal{F}_{n(v)}^{\theta}(\check{U}) .
$$

Recall that $V$ is finite dimensional. Hence the set $\left\{n(v) \mid v \in v_{\mu}^{B}+V\right\}$ has a minimal element $n_{\min }$. In what follows we will construct an element $w \in v_{\mu}^{B}+V$ such that $w-b(w) \in \mathcal{F}_{n_{\min }-1 / N}(\check{U})$ leading our assumption $\left(v_{\mu}^{B}+V\right) \cap \check{B} \neq\{0\}$ to a contradiction.

Claim 1: $n_{\min }<h^{\Sigma}(2 \mu)$.

Proof of Claim 1. Recall that $Y^{\mu} \in G^{-} \mathcal{M}^{+} T_{\Theta}^{\prime}$ is a vector of weight $2 \tilde{\mu}$ with respect to the right adjoint action. Hence, we can write

$$
Y^{\mu} \in \sum_{\tilde{\beta}=2 \tilde{\mu}} G_{\beta}^{-} \mathcal{M}^{+} T_{\Theta}^{\prime} .
$$

Using Lemma 5.1 and induction one obtains

$$
\begin{aligned}
Y^{\mu} & \in \sum_{h^{\Sigma}(\operatorname{wt}(I))=h^{\Sigma}(2 \mu)} B_{I} \mathcal{M} T_{\Theta}^{\prime}+\sum_{h^{\Sigma}(\operatorname{wt}(J))<h^{\Sigma}(2 \mu)} B_{J} G^{+} \check{U}^{0} \\
& \subseteq \sum_{h^{\Sigma}(\operatorname{wt}(I))=h^{\Sigma}(2 \mu)} B_{I} \mathcal{M} T_{\Theta}^{\prime}+\sum_{h^{\Sigma}(\eta)<h^{\Sigma}(2 \mu)} U_{-\eta}^{-} G^{+} \check{U}^{0} .
\end{aligned}
$$

It follows from the definition (41) of $v_{\mu}^{B}$ that

$$
v_{\mu}^{B} \in \sum_{h^{\Sigma}(\operatorname{wt}(I))=h^{\Sigma}(2 \mu)} B_{I} \mathcal{M} T_{\Theta}^{\prime}+\sum_{h^{\Sigma}(\eta)<h^{\Sigma}(2 \mu)} \check{U}_{-\eta} .
$$

By Proposition 6.3(iii), applying the projection $b$ to both sides of (53) yields

$$
v_{\mu}^{B}-b\left(v_{\mu}^{B}\right) \in \sum_{h^{\Sigma}(\eta)<h^{\Sigma}(2 \mu)} \check{U}_{-\eta} .
$$

Hence we can apply Lemma 8.2 to $v_{\mu}^{B}$ and there exists $w \in v_{\mu}^{B}+V$ with $n(w)<$ $h^{\Sigma}(2 \mu)$. Therefore, $n_{\min }<h^{\Sigma}(2 \mu)$.

We now continue with the proof of Theorem 8.3. Consider $v \in v_{\mu}^{B}+V$ such that $n(v)=n_{\min }$. By definition of $V$ we can write $v=v_{\mu}^{B}+v^{\prime}+v^{\prime \prime}$ where

$$
v^{\prime} \in \sum_{\substack{\eta \leq \mu \\ h^{\Sigma}(2 \eta)>n_{\min }}}\left(\left(\operatorname{ad}_{r} U\right) \tau(2 \eta)\right)^{B}, \quad v^{\prime \prime} \in \sum_{\substack{\eta \leq \mu \\ h^{\Sigma}(2 \eta) \leq n_{\min }}}\left(\left(\operatorname{ad}_{r} U\right) \tau(2 \eta)\right)^{B} .
$$

It follows from Proposition 6.3 (ii) that $v^{\prime \prime}-b\left(v^{\prime \prime}\right) \in \mathcal{F}_{n_{\text {min }}}^{\theta}(\check{U})$. Hence subtracting $v^{\prime \prime}$ we may assume that $v=v_{\mu}^{B}+v^{\prime}$ and $n(v)=n_{\min }$. 
By Lemma 4.1, we see that $b(v) \in G^{-} U^{+} T_{\geq}$. It follows from Corollary 4.3 that $v \in G^{-} U^{+} T_{\geq}$. The definition of the degree function defining $\mathcal{F}^{\theta}$ implies that

$$
\begin{aligned}
& v-b(v) \in \sum_{\eta \geq 0} \sum_{\beta \geq 0} \sum_{\tilde{\gamma} \geq r} \sum_{h^{\Sigma}(\eta+\beta+\gamma) \leq n_{\min }}\left(G_{-\eta}^{-} U_{\beta}^{+}\right) \tau(\gamma) \\
& \subseteq \sum_{h^{\Sigma}(\eta)=n_{\min }} G_{-\eta}^{-} \mathcal{M}^{+} T_{\Theta}^{\prime}+\sum_{h^{\Sigma}(\eta)<n_{\min }}\left(G_{-\eta}^{-} U^{+}\right) T_{\geq} .
\end{aligned}
$$

Applying Lemma 5.1 and induction to (55) yields

$$
v-b(v) \in \sum_{h^{\Sigma}(\operatorname{wt}(I))=n_{\min }} B_{I} \mathcal{M}^{+} T_{\Theta}^{\prime}+\sum_{h^{\Sigma}(\operatorname{wt}(I))<n_{\min }} B_{I} U^{+} \check{U}^{0} .
$$

By the definition of the projection map $b$, we have that $v-b(v) \in B \check{T}_{\Theta} \mathcal{G}$. Hence the definition of $\mathcal{G}$ in Section 6.1 combined with (56) imply that

$$
v-b(v) \in \sum_{h^{\Sigma}(\operatorname{wt}(I))<n_{\min }} B_{I} U^{+} \check{U}^{0} .
$$

Hence by Lemma 5.1, we see that

$$
v-b(v) \in \sum_{h^{\Sigma}(\eta)<n_{\min }} G_{-\eta}^{-} U^{+} \check{U}^{0} \subseteq \sum_{h^{\Sigma}(\eta)<n_{\min }} \check{U}_{-\eta} .
$$

Assertion (57) allows us to apply Lemma 8.2 to $v$. Thus there exists $w \in v_{\mu}^{B}+V$ such that $n(w) \leq n_{\min }-\frac{1}{N}$. This is the desired contradiction to the minimality of $n_{\min }$.

8.4. Central elements in $\left(\operatorname{ad}_{r} U\right) \tau(2 \mu)$. We show here that if $\mu \in P_{Z(\check{B})}$ satisfies a suitable minimality condition, then Theorem 8.3 yields elements in $Z(\check{B}) \cap$ $\left(\operatorname{ad}_{r} U\right) \tau(2 \mu)$. These elements are used in [Kol] to obtain solutions of the reflection equation and hence further establish the link between different approaches to the construction of quantum symmetric pairs.

Corollary 8.4 Fix $\mu \in P_{Z(\check{B})}$. Assume that for all $\nu \in P(\pi)$ the condition $\nu<\mu$ implies $\nu \notin P^{+}(\pi) \backslash\{0\}$. Then there exists a nonzero element $d_{\mu} \in Z(\check{B}) \cap$ $\left(\operatorname{ad}_{r} U\right) \tau(2 \mu)$.

${ }^{1}$ Note added in proof: At the time the paper was accepted for publication the authors had become aware of the following straightforward consequence of Theorem 8.3 which implies in particular that the minimality condition in Corollary 8.4 can be dropped.

Theorem. For any $\mu \in P^{+}(\pi)$ one has

$$
\operatorname{dim}\left(Z(\check{B}) \cap\left(\operatorname{ad}_{r} U\right) \tau(2 \mu)\right)= \begin{cases}1 & \text { if } \mu \in P_{Z(\check{B})}, \\ 0 & \text { else. }\end{cases}
$$

Proof. Let $p_{\mu}: F_{r}(\check{U}) \rightarrow\left(\operatorname{ad}_{r} U\right) \tau(2 \mu)$ denote the projection map with respect to the direct sum decomposition (9). Note that $p_{\mu}$ is a homomorphism of right $\check{U}$-comodules. As $\check{B}$ is a left coideal subalgebra of $\breve{U}$ one obtains

$$
p_{\mu}\left(\check{B} \cap F_{r}(\check{U})\right) \subseteq \check{B} .
$$

If $\mu \in P_{Z(\check{B})}$, then one gets in particular for the element $d_{\mu}$ from Theorem 8.3 the relation $p_{\mu}\left(d_{\mu}\right) \in Z(\breve{B}) \cap\left(\operatorname{ad}_{r} \breve{U}\right) \tau(2 \mu)$. 
Proof. The definition of $\left(V_{0}^{\mu}\right)^{B}$ in Section 7.3 and Theorem 1.6 imply

$$
\left(V_{0}^{\mu}\right)^{B} \subseteq \sum_{\nu \in P^{+}(\pi), \nu \leq \mu}\left(\operatorname{ad}_{r} U\right) \tau(2 \nu) .
$$

The assumption about $\mu$ now implies that $\left(V_{0}^{\mu}\right)^{B}$ is a subspace of $\left(\operatorname{ad}_{r} U\right) \tau(2 \mu)+$ $\left(\operatorname{ad}_{r} U\right) \tau(0)$.

8.5. A basis for the center of $\check{B}$. By Theorem 8.3 , we can choose an element $d_{\mu} \in Z(\check{B})$ which lies in $v_{\mu}^{B}+\left(V_{0}^{\mu}\right)^{B}$ for each $\mu \in P_{Z(\check{B})}$. We will assume these elements to be fixed for the rest of this paper. Note that when $\mu=0$, then $d_{0}=v_{0}^{B}=Y_{0}$ is a nonzero scalar.

Theorem 8.5. The set $\left\{d_{\mu} \mid \mu \in P_{Z(\check{B})}\right\}$ is a basis of the vector space $Z(\check{B})$.

Proof. Note that $d_{\mu}$ lies in $\left(\operatorname{ad}_{r} U\right) \tau(2 \mu) \backslash\{0\}$ up to terms of lower filter degree with respect to $\mathcal{F}$. Hence the set $\left\{d_{\mu} \mid \mu \in P_{Z(\breve{B})}\right\}$ is linearly independent.

Let $C$ be in $Z(\check{B})$. Write $C=\sum_{\beta} c_{\beta}$ with $c_{\beta} \in \check{U}_{-\beta}$. Set

$$
m=\max \left\{h^{\Sigma}(\beta) \mid c_{\beta} \neq 0\right\} .
$$

Let $\left\{\beta_{i} \mid 1 \leq i \leq k\right\}$ denote the set of weights such that $h^{\Sigma}\left(\beta_{i}\right)=m$ and $c_{\beta_{i}} \neq 0$. Since $C$ is a sum of spherical vectors inside $F_{r}(\check{U})$, it follows from (11) that each $c_{\beta_{i}}$ is a highest weight vector with respect to the right adjoint action contained in a finite-dimensional spherical submodule of $F_{r}(\check{U})$. By (8) and the fact that $C \in \check{B}$, we can write

$$
C \in \sum_{I \in \mathcal{I}} B_{I} \mathcal{M}^{+} T_{\Theta}^{\prime}
$$

It follows from Lemma 5.1, that $c_{\beta_{i}} \in G^{-} \mathcal{M}^{+} T_{\Theta}^{\prime}$ for $i=1, \ldots, k$. By Theorem 7.2 , there exist scalars $a_{i \gamma}$ such that

$$
c_{\beta_{i}}=\sum_{\gamma \in P_{Z(\check{B})}, h^{\Sigma}(\gamma)=m} a_{i \gamma} Y_{\gamma} .
$$

Note that the fact that each $\beta_{i}$ is a spherical weight forces $m=h^{\Sigma}\left(\beta_{i}\right) \geq 0$. If $m=0$, then $i=1, \beta_{1}=0$, and $c_{0}$ is just a scalar multiple of the nonzero scalar $Y_{0}$. Moreover, the simple $\left(\operatorname{ad}_{r} U\right)$-module generated by $c_{0}$ is just $\mathcal{C}=\mathcal{C} d_{0}$, so $C \in \mathcal{C} d_{0}$. Assume $m>0$. By Theorem 8.3 .

$$
C-\sum_{i=1}^{k} \sum_{h^{\Sigma}(\gamma)=m} a_{i \gamma} d_{\gamma} \in Z(\check{B}) \cap \sum_{h^{\Sigma}(\beta)<m} \check{U}_{-\beta} .
$$

The theorem follows using induction on $m$.

\section{Realizing $Z(\check{B})$ as a polynomial Ring}

We now calculate the set $P_{Z(\check{B})}$ explicitly. It turns out that $P_{Z(\check{B})}$ is closely related to the rank of the invariant Lie algebra $\mathfrak{g}^{\theta}$. This is used to show that $Z(\check{B})$ is a polynomial ring in $\operatorname{rank}\left(\mathfrak{g}^{\theta}\right)$ variables. To this end it is essential that central elements in $G(\leq 2 \mu)$ for $\mu \in P_{Z(\breve{B})}$ are unique up to elements of lower filter degree. 
9.1. Determining $P_{Z(\breve{B})}$. We restrict to the case of irreducible symmetric pairs. By [Ara62, 2.5 and 5.1], the pair $\left(\mathfrak{g}, \mathfrak{g}^{\theta}\right)$ is irreducible if and only if $\mathfrak{g}$ is simple, or $\mathfrak{g}=\mathfrak{g}^{\prime} \oplus \mathfrak{g}^{\prime}$ for some simple $\mathfrak{g}^{\prime}$ and $\theta$ interchanges the two isomorphic components.

In the second case the corresponding quantum symmetric pair $\check{U}, \check{B}$ is given explicitly in [Let03, Section 7] and it is straightforward to check that $\check{B} \cong \check{U}_{q}\left(\mathfrak{g}^{\prime}\right)$ as $\mathcal{C}$-algebras. Hence the center $Z(\check{B}) \cong Z\left(\check{U}_{q}\left(\mathfrak{g}^{\prime}\right)\right)$ is well known by [JL94]. Here we obtain the same result, it should, however, be borne in mind that our calculations heavily depended on the previous knowledge of the structure of $F_{r}(\check{U})$. As $\pi_{\Theta}$ is empty one gets $P_{Z(\check{B})}=\left\{\mu \in P^{+}(\pi) \mid \Theta(\mu)=-w_{0} \mu\right\}$. Let $P^{+}\left(\pi^{\prime}\right)$ denote the dominant integral weights of $\mathfrak{g}^{\prime}$ with respect to a set of simple roots $\pi^{\prime}$ for $\mathfrak{g}^{\prime}$. Without loss of generality, we can assume that $P^{+}(\pi)=P^{+}\left(\pi^{\prime}\right) \times P^{+}\left(\pi^{\prime}\right)$. Then

$$
P_{Z(\check{B})}=\left\{\left(\mu,-w_{0}^{1} \mu\right) \mid \mu \in P^{+}\left(\pi^{\prime}\right)\right\}
$$

where $w_{0}^{1}$ denotes the longest element in the Weyl group of $\mathfrak{g}^{\prime}$.

Recall Araki's list [Ara62, 5.11] of all symmetric pairs $\left(\mathfrak{g}, \mathfrak{g}^{\theta}\right)$ for simple $\mathfrak{g}$. Following [Let03, Section 7] the parameter $p$ occurring in Araki's list will here be denoted by $r$, and as before $n=\operatorname{rank}(\mathfrak{g})$.

Proposition 9.1. Assume that $\left(\mathfrak{g}, \mathfrak{g}^{\theta}\right)$ is an irreducible symmetric pair. The subset $P_{Z(\check{B})}$ of $P^{+}(\pi)$ is determined by the following list.

1) If $\mathfrak{g}=\mathfrak{g}^{\prime} \oplus \mathfrak{g}^{\prime}$ and $P^{+}(\pi)=P^{+}\left(\pi^{\prime}\right) \times P^{+}\left(\pi^{\prime}\right)$, then $P_{Z(\check{B})}=\left\{\left(\mu,-w_{0}^{1} \mu\right) \mid \mu \in\right.$ $\left.P^{+}\left(\pi^{\prime}\right)\right\}$.

2) If $\mathfrak{g}$ is one of $B_{n}, C_{n}, E_{7}, E_{8}, F_{4}$, or $G_{2}$, then $P_{Z(\check{B})}=P^{+}(\pi)$.

3) If $\left(\mathfrak{g}, \mathfrak{g}^{\theta}\right)$ is of type AI, AII, (DI, case 3), (DIII, case 1), EI, or EIV, then $P_{Z(\check{B})}=\left\{\mu \in P^{+}(\pi) \mid \mu=-w_{0} \mu\right\}$.

4) If $\left(\mathfrak{g}, \mathfrak{g}^{\theta}\right)$ is of type AIII, AIV, EII, EIII, or (DIII, case 2), then $P_{Z(\check{B})}=P^{+}(\pi)$.

5) If $\left(\mathfrak{g}, \mathfrak{g}^{\theta}\right)$ is of type (DI, case 1$)$ or DII, then

$$
P_{Z(\check{B})}= \begin{cases}P^{+}(\pi) & \text { if } r \text { is even, } \\ \sum_{i=1}^{n-2} \mathbb{N} \omega_{i}+\mathbb{N}\left(\omega_{n-1}+\omega_{n}\right) & \text { if } r \text { is odd. }\end{cases}
$$

6) If $\left(\mathfrak{g}, \mathfrak{g}^{\theta}\right)$ is of type (DI, case 2$)$, then

$$
P_{Z(\breve{B})}= \begin{cases}P^{+}(\pi) & \text { if } n \text { is odd, } \\ \sum_{i=1}^{n-2} \mathbb{N} \omega_{i}+\mathbb{N}\left(\omega_{n-1}+\omega_{n}\right) & \text { if } n \text { is even. }\end{cases}
$$

Proof. The diagonal case 1) has already been handled above. Consider now the case where $\mathfrak{g}$ is simple. Recall [Let02, Section 7] that $\Theta(\mu)=-w_{0}^{\prime} d(\mu)$ where $d$ is a diagram automorphism. Hence, if $d=\mathrm{id}$, then $P_{Z(\check{B})}$ consists of all selfdual dominant integral weights. This proves statements 2) and 3) of the proposition.

Note that for all cases listed in 4$)$ as well as $(D I$, case $1, r$ even) and (DI, case $2, n$ odd) one has $d=-w_{0}$. Note, moreover, that $\alpha_{i}-d\left(\alpha_{i}\right)$ is invariant under $\Theta$ for any $\alpha_{i} \in \pi$. Hence one obtains

$$
\Theta\left(\alpha_{i}\right)-\alpha_{i}=\Theta\left(d\left(\alpha_{i}\right)\right)-d\left(\alpha_{i}\right)=-w_{0}^{\prime} \alpha_{i}+w_{0} \alpha_{i}
$$

for any $\alpha_{i} \in \pi$. This proves 4), 5) for $r$ even, and 6) for $n$ odd. 
The claim of 5) follows from $d\left(\omega_{i}\right)=-w_{0}^{\prime} \omega_{i}=\omega_{i}$ for $i=1, \ldots, n-2$ and

$$
\begin{aligned}
d\left(\omega_{n-1}\right) & = \begin{cases}\omega_{n-1} & \text { if } n-r \text { even, } \\
\omega_{n} & \text { if } n-r \text { odd },\end{cases} \\
d\left(\omega_{n}\right) & = \begin{cases}\omega_{n} & \text { if } n-r \text { even, } \\
\omega_{n-1} & \text { if } n-r \text { odd },\end{cases} \\
-w_{0}^{\prime}\left(\omega_{n-1}\right) & = \begin{cases}\omega_{n-1}-\omega_{r} & \text { if } n-r \text { even, } \\
\omega_{n}-\omega_{r} & \text { if } n-r \text { odd },\end{cases} \\
-w_{0}^{\prime}\left(\omega_{n}\right) & = \begin{cases}\omega_{n}-\omega_{r} & \text { if } n-r \text { even, } \\
\omega_{n-1}-\omega_{r} & \text { if } n-r \text { odd } .\end{cases}
\end{aligned}
$$

Note that for the symmetric pair of type $(D I$, case 2$)$ one has $w_{0}^{\prime}=$ id and hence $\mu \in P_{Z(\check{B})}$ if and only if $d(\mu)=-w_{0} \mu$. This condition holds precisely for all dominant integral weights given in 6$)$.

9.2. The rank of $\mathfrak{g}^{\theta}$. Note that for every $\left(\mathfrak{g}, \mathfrak{g}^{\theta}\right)$ there exist (up to ordering) uniquely determined elements $\nu_{1}, \ldots, \nu_{m} \in P^{+}(\pi)$ such that

$$
P_{Z(\check{B})}=\bigoplus_{i=1}^{m} \mathbb{N} \nu_{i}
$$

We define $\operatorname{rank}\left(P_{Z(\check{B})}\right):=m$. Moreover, let $\operatorname{rank}\left(\mathfrak{g}^{\theta}\right)$ denote the rank of the reductive Lie algebra $\mathfrak{g}^{\theta}$.

Proposition 9.2. The relation $\operatorname{rank}\left(P_{Z(\check{B})}\right)=\operatorname{rank}\left(\mathfrak{g}^{\theta}\right)$ holds.

Proof. We may restrict to the case where $\mathfrak{g}$ is simple. By Hel78, Chapter X, Thm. 5.15 (ii)] for every $\left(\mathfrak{g}, \mathfrak{g}^{\theta}\right)$ there exists an automorphism $\nu$ of the Dynkin diagram of $\mathfrak{g}$ such that $\operatorname{rank}\left(\mathfrak{g}^{\theta}\right)$ coincides with the dimension of the fixed point set $\mathfrak{h}^{\nu}$ in the corresponding Cartan subalgebra $\mathfrak{h}$ of $\mathfrak{g}$. The order $k$ of $\nu$ can be seen in [Hel78, p. 514, Tables II and III]. In particular, if $\mathfrak{g}$ has no nontrivial diagram automorphism one gets $\operatorname{rank}\left(\mathfrak{g}^{\theta}\right)=\operatorname{rank}(\mathfrak{g})$. This proves the proposition if $\mathfrak{g}$ is of type $B_{n}, C_{n}, E_{7}, E_{8}, F_{4}$, or $G_{2}$. The other cases follow by inspection from Hel78, p. 514, Tables II and III] and Proposition 9.1. For the convenience of the reader we collect the order $k$ of the diagram automorphism $\nu$ for the remaining cases:

$k=1: A I I I, A I V,(D I$, case $1, r$ even $),(D I$, case $2, n$ odd $),(D I$, case $3, n$ even), DIII, EII, EIII.

$\underline{k=2:} A I, A I I,(D I$, case $1, r$ odd $),(D I$, case $2, n$ even $),(D I$, case $3, n$ odd), $D I I, E I, E I V$.

9.3. $Z(\check{B})$ is a polynomial ring in $\operatorname{rank}\left(\mathfrak{g}^{\theta}\right)$ variables. Recall from (59) and Proposition 9.2 that $P_{Z(\breve{B})}$ is generated over $\mathbb{N}$ by $m=\operatorname{rank}\left(\mathfrak{g}^{\theta}\right)$ linearly independent elements $\nu_{1}, \ldots, \nu_{m} \in P^{+}(\pi)$. Let $d_{\nu_{1}}, \ldots, d_{\nu_{m}}$ denote the corresponding central elements as chosen at the beginning of Section 8.4. Let $\mathcal{C}\left[z_{1}, \ldots, z_{m}\right]$ be the polynomial ring in $m$ generators.

Theorem 9.3. The algebra homomorphism $\Phi: \mathcal{C}\left[z_{1}, \ldots, z_{m}\right] \rightarrow Z(\check{B})$ defined by $\Phi\left(z_{i}\right)=d_{\nu_{i}}$ is an isomorphism. 
Proof. Recall from Theorem 8.3 that in the graded algebra Gr the element $\overline{d_{\mu}}$ represented by $d_{\mu}$ lies in $\left(\operatorname{ad}_{r} U\right) \overline{\tau(2 \mu)}$. Recall, moreover, that for $\mu, \eta \in P_{Z(\check{B})}$ one has $\overline{d_{\mu}} \overline{d_{\eta}} \in\left(\operatorname{ad}_{r} U\right) \overline{\tau(\mu+\eta)}$ [JL94, Prop. 4.12 (iii)] and that $\mathrm{Gr}$ is an integral domain [JL94, Prop. 4.12 (iii)]. Hence $d_{\mu} d_{\nu}$ is a central element in $\check{B}$ which consists of a nonzero component in $\left(\operatorname{ad}_{r} U\right) \tau(\mu+\nu)$ and terms of lower filter degree. By Theorem 8.5 one gets $d_{\mu} d_{\eta}=a_{\mu \eta} d_{\mu+\eta}$ for some constant $a_{\mu \eta} \in \mathcal{C}$ up to terms of lower filter degree in $Z(\check{B})$. Hence up to terms of lower filter degree in $Z(\check{B})$ any element of $Z(\check{B})$ can be written as a polynomial in the generators $d_{\nu_{1}}, \ldots, d_{\nu_{m}}$. By induction this proves that $\Phi$ is surjective.

As $\mathrm{Gr}$ is an integral domain, the monomial $d_{\nu_{1}}^{n_{1}} \ldots d_{\nu_{m}}^{n_{m}}$ represents a nonzero element in $\left(\operatorname{ad}_{r} U\right) \overline{\tau\left(2 \sum n_{i} \nu_{i}\right)}$. Hence $\Phi$ is injective.

\section{Appendix: Commonly USED nOtation}

Section 1.1:

$\begin{array}{ll}\mathbb{C} & \text { complex numbers } \\ \mathbb{Z} & \text { integers } \\ \mathbb{N} & \text { nonnegative integers } \\ \mathfrak{g} & \text { finite-dimensional complex semisimple Lie algebra } \\ \mathfrak{h} & \text { Cartan subalgebra of } \mathfrak{g} \\ \Delta & \text { root system of } \mathfrak{g}, \mathfrak{h} \\ \pi & \text { set }\left\{\alpha_{1}, \ldots, \alpha_{n}\right\} \text { of simple roots for } \Delta \\ (\cdot, \cdot) & \text { Cartan inner product on } \mathfrak{h}^{*} \\ W & \text { Weyl group of } \Delta \\ w_{0} & \text { longest element in } W \text { with respect to } \pi \\ Q(\pi) & \text { root lattice of } \Delta \\ P(\pi) & \text { weight lattice of } \Delta \\ Q^{+}(\pi) & \mathbb{N} \pi \\ P^{+}(\pi) & \text { dominant integral weights associated to } \pi \\ \omega_{1} \ldots, \omega_{n} & \text { fundamental weights } \\ \leq & \text { standard partial ordering on } \mathfrak{h}^{*}\end{array}$

Section 1.2:

$\begin{array}{ll}q & \text { an indeterminate } \\ \mathcal{C} & \mathbb{C}(q) \text { or } \mathbb{C}\left(q^{\frac{1}{2}}\right) \\ U_{q}(\mathfrak{g}) & \text { quantized enveloping algebra of } \mathfrak{g} \\ t_{i}, x_{i}, y_{i} & \text { generators of } U_{q}(\mathfrak{g}) \\ U & U_{q}(\mathfrak{g}) \\ \Delta & \text { coproduct of } U_{q}(\mathfrak{g}) \\ \sigma & \text { antipode of } U_{q}(\mathfrak{g}) \\ \epsilon & \text { counit of } U_{q}(\mathfrak{g}) \\ \operatorname{ad}_{r} & \text { right adjoint action } \\ U^{+} & \text {subalgebra of } U \text { generated by } x_{1}, \ldots, x_{n} \\ U^{-} & \text {subalgebra of } U \text { generated by } y_{1}, \ldots, y_{n} \\ G^{+} & \text {subalgebra of } U \text { generated by } x_{1} t_{1}^{-1}, \ldots, x_{n} t_{n}^{-1} \\ G^{-} & \text {subalgebra of } U \text { generated by } y_{1} t_{1}, \ldots, y_{n} t_{n} \\ T & \text { group generated by } t_{1}^{ \pm 1}, \ldots, t_{n}^{ \pm 1} \\ U^{0} & \text { subalgebra of } U \text { generated by } T\end{array}$


$\tau$

$\check{T}$

$\check{U}$

$\check{U}^{0}$

$(y t)_{I}$

$\mathrm{wt}(I)$

$\mathcal{I}$

Section 1.3:

$S_{\mu}$

$V(\mu)$

$V(\mu)^{*}$

Section 1.4:

$\theta$

$\mathfrak{g}^{\theta}$

$\Theta$

$\pi_{\Theta}$

$p$

$\pi^{*}$

$\tilde{\alpha}$

$\Sigma$

$P(\Sigma)$

$P^{+}(\Sigma)$

$\geq r$

Section 1.5:

$\mathcal{M}$

$T_{\Theta}$

$B$

$B_{i}, \tilde{\theta}\left(y_{i}\right)$

$B_{i}$

$\mathcal{M}^{+}$

$B_{I}$

$T_{\Theta}^{\prime}$

$\check{B}$

Section 1.6:

$F_{r}(\check{U})$

$Z(\check{B})$

Section 1.7:

$M^{B}$ spherical weight

set of $B$-invariant elements in $M$

weight in $2 P^{+}(\Sigma)$

Section 2.1:

$h$

$\mathcal{F}$

$\mu$ weight space of $S$

$\{x \in \mathfrak{g} \mid \theta(x)=x\}$

involution of $\mathfrak{h}^{*}$ induced by $\theta$

$\left\{\alpha_{i} \in \pi \mid \Theta\left(\alpha_{i}\right)=\alpha_{i}\right\}$

$(\alpha-\Theta(\alpha)) / 2$

weight lattice of $\Sigma$

$\{\tau(\lambda) \mid \lambda \in Q(\pi)$ and $\Theta(\lambda)=\lambda\}$

for $\alpha_{i} \notin \pi_{\Theta}$, see (7)

$y_{i} t_{i}$ if $\alpha_{i} \in \pi_{\Theta}$

$\mathcal{M} \cap U^{+}$

$\{\tau(\lambda) \mid \lambda \in P(\pi)$ and $\Theta(\lambda)=\lambda\}$

$\left\{a \in \check{U} \mid \operatorname{dim}\left(\left(\operatorname{ad}_{r} U\right) a\right)<\infty\right\}$

center of $\check{B}$ group isomorphism from $Q(\pi)$ onto $T$

extension of $T$ isomorphic to $P(\pi)$

simply connected quantized enveloping algebra of $\mathfrak{g}$

subalgebra of $\check{U}$ generated by $\tau(\lambda), \lambda \in P(\pi)$

$y_{i_{1}} t_{i_{1}} \ldots y_{i_{m}} t_{i_{m}}$ where $I=\left(i_{1}, \ldots, i_{m}\right)$

$\alpha_{i_{1}}+\cdots+\alpha_{i_{m}}$ where $I=\left(i_{1}, \ldots, i_{m}\right)$

set of multi-indices such that $\left\{(y t)_{I} \mid I \in \mathcal{I}\right\}$ is a basis for $G^{-}$.

simple left $U$-module of highest weight $\mu$

dual space of $V(\mu)$ with natural right $U$-module structure

involutive Lie algebra automorphism of $\mathfrak{g}$

permutation on $\{1, \ldots, n\}$ satisfying (6)

$\left\{\alpha_{i} \in \pi \backslash \pi_{\Theta} \mid i \leq p(i)\right\}$

restricted root system $\{\tilde{\alpha} \mid \alpha \in \Delta, \Theta(\alpha) \neq \alpha\}$

set of dominant integral weights inside $P(\Sigma)$

standard partial ordering associated to restricted root system

algebra generated by $x_{i}, y_{i}, t_{i}^{ \pm 1}$, for $\alpha_{i} \in \pi_{\Theta}$

algebra generated by $\mathcal{M}, T_{\Theta}, B_{i}$, for $\alpha_{i} \in \pi \backslash \pi_{\Theta}$

$B_{i_{1}} \ldots B_{i_{m}}$ where $I=\left(i_{1}, \ldots, i_{m}\right)$

subalgebra of $\check{U}$ generated by $B$ and $T_{\Theta}^{\prime}$

height function on $\mathbb{Q} \pi$ defined by $(12)$

$\frac{1}{N} \mathbb{Z}$-filtration defined by degree function (13) 


$\begin{array}{ll}\mathrm{Gr} & \operatorname{gr}_{\mathcal{F}}(\check{U}) \\ \mathrm{Gr}^{0} & \operatorname{gr}_{\mathcal{F}}\left(\check{U}^{0}\right) \\ \mathrm{Gr}^{-} & \operatorname{gr}_{\mathcal{F}}\left(U^{-}\right) \\ \mathrm{Gr}^{+} & \operatorname{gr}_{\mathcal{F}}\left(G^{+}\right) \\ \bar{a} & \text { graded image of } a \text { in } \mathrm{Gr}\end{array}$

Section 2.2:

$K(2 \lambda)^{-}$

$K(2 \lambda)^{+}$

$F_{r}(\mathrm{Gr})$

$g$

subspace of $\mathrm{Gr}^{-}$such that $\left(\operatorname{ad}_{r} U^{-}\right) \overline{\tau(2 \lambda)}=K(2 \lambda)^{-} \otimes \mathcal{C} \overline{\tau(2 \lambda)}$

subspace of $\mathrm{Gr}^{+}$such that $\left(\operatorname{ad}_{r} U^{+}\right) \overline{\tau(2 \lambda)}=\overline{\mathcal{C}} \overline{\tau(2 \lambda)} \otimes K(2 \lambda)^{+}$

$\left\{a \in \mathrm{Gr} \mid \operatorname{dim}\left(\left(\operatorname{ad}_{r} U\right) a\right)<\infty\right\}$

isomorphism of $F_{r}(\check{U})$ onto $F_{r}(\mathrm{Gr})$ given by (17)

Section 2.3:

$h^{\Sigma}$

$\mathcal{F}^{\theta}$

$\mathrm{Gr}^{\theta}$

$\bar{a}^{\theta}$

height function associated to $\Sigma$ defined by (19)

$\frac{1}{N} \mathbb{Z}$-filtration defined by degree function (20)

$\operatorname{gr}_{\mathcal{F}}^{\theta}(\check{U})$

graded image in $\mathrm{Gr}^{\theta}$ of $a \in \check{U}$

Section 2.4:

$F_{r}\left(\mathrm{Gr}^{\theta}\right)$

$g^{\theta}$

$\left\{a \in \operatorname{Gr}^{\theta} \mid \operatorname{dim}\left(\left(\operatorname{ad}_{r} U\right) a\right)<\infty\right\}$

isomorphism of $F_{r}(\check{U})$ onto $F_{r}\left(\mathrm{Gr}^{\theta}\right)$ given in Proposition 2.4

Section 3.2:

$W\left(\pi_{\Theta}\right)$

$w_{0}^{\prime}$

$\mathfrak{m}$

Weyl group for root system of $\pi_{\Theta}$

longest element in $W\left(\pi_{\Theta}\right)$

semisimple Lie subalgebra of $\mathfrak{g}$ with simple roots $\pi_{\Theta}$

Section 4.1:

$T_{\geq}$

$\operatorname{span}\left\{\tau(\delta) \mid \delta \in P(\pi)\right.$ and $\left.\tilde{\delta} \geq_{r} 0\right\}$

Section 4.2:

$G(\leq \mu)$

$U^{-} G^{+} \tau(\mu) \mathcal{C}\left[t_{1}^{-2}, \ldots, t_{n}^{-2}\right]$

Section 5.1:

$\check{\mathcal{A}}$

$\check{T}_{\Theta}$

ad

$N^{+}$

$\{\tau(\tilde{\alpha}) \mid \alpha \in P(\pi)\}$

$\{\tau((\alpha+\Theta(\alpha)) / 2) \mid \alpha \in P(\pi)\}$

left adjoint action

subalgebra of $U^{+}$generated by $\left(\operatorname{ad} \mathcal{M}^{+}\right)\left(\mathcal{C}\left[x_{i} \mid \alpha_{i} \notin \pi_{\Theta}\right]\right)$

Section 6.1:

$\mathrm{N}_{+}^{+}$

$\mathcal{A}^{+}$

$\mathcal{G}$

intersection of $N^{+}$with augmentation ideal of $U$

$\check{\mathcal{A}} \backslash\{1\}$

$\check{\mathcal{A}} N_{+}^{+}+\check{\mathcal{A}}^{+}$

Section 6.2:

b

projection of $\check{U} \check{T}_{\Theta}$ onto $\check{B}_{\Theta}$ defined by (33) 
Section 7:

$\mathcal{V}$

$$
G^{-} \mathcal{M}^{+} T_{\Theta}^{\prime} \cap F_{r}(\check{U})^{U^{-}} \cap \sum_{\lambda \in 2 P^{+}(\Sigma)} \check{U}_{-\lambda}
$$

Section 7.1:

$P_{Z(\check{B})} \quad\left\{\mu \in P^{+}(\pi) \mid \Theta(\mu)=\mu+w_{0} \mu-w_{0}^{\prime} \mu\right\}$

Section 7.2:

$Y^{\mu}$

nonzero vector in $\mathcal{V} \cap\left(\operatorname{ad}_{r} U\right) \tau(2 \mu)$ of weight $2 \tilde{\mu}, \quad \mu \in P_{Z(\check{B})}$

Section 7.3:

$v_{\mu}^{B}$

element in $\left(\left(\operatorname{ad}_{r} U\right) Y^{\mu}\right)^{B}$ defined by (41)

$V_{0}^{\mu}$

sum of simple spherical modules $V$ in $G(\leq 2 \mu)$ with $V \neq V(2 \tilde{\mu})^{*}$

Section 8.4:

$d_{\mu}$

element in $Z(\check{B}) \cap\left(v_{\mu}^{B}+\left(V_{0}^{\mu}\right)^{B}\right)$, for $\mu \in P_{Z(\check{B})}$

Section 9.2:

$\nu_{1}, \ldots, \nu_{m} \quad$ linearly independent generators for $P_{Z(\breve{B})}($ see $(59))$

$\operatorname{rank}\left(P_{Z(\check{B})}\right) \quad m$ defined by (59)

\section{REFERENCES}

[Ara62] S. Araki, On root systems and an infinitesimal classification of irreducible symmetric spaces, J. Math. Osaka City Univ. 13 (1962), 1-34. MR0153782 (27:3743)

[CK90] C. De Concini and V.G. Kac, Representations of quantum groups at roots of 1, Operator algebras, unitary representations, enveloping algebras and invariant theory (A. Connes, M. Duflo, A. Joseph, and R. Rentschler, eds.), Birkhäuser, 1990, pp. 471506. MR 1103601 (92g:17012)

[Dij96] M.S. Dijkhuizen, Some remarks on the construction of quantum symmetric spaces, Acta Appl. Math. 44 (1996), no. 1-2, 59-80. MR1407040 (98c:33020)

[DN98] M.S. Dijkhuizen and M. Noumi, A family of quantum projective spaces and related $q-$ hypergeometric orthogonal polynomials, Transactions of the AMS 350 (1998), 3269-3296. MR:1432197 (2000m:33023)

[DS99] M.S. Dijkhuizen and J.V. Stokman, Some limit transitions between BC type orthogonal polynomials interpreted on quantum complex Grassmannians, Publ. Res. Inst. Math. Sci. 35 (1999), 451-500. MR.1710751 (2001b:33023)

[FM98] F. Fauquant-Millet, Sur une algèbre parabolique $\mathbf{P}$ de $\check{U}_{q}\left(\mathbf{s l}_{n+1}\right)$ et ses semi-invariants par l'action adjointe de P, Bull. Sci. Math. 122 (1998), 495-519. MR.1653466 (99k:17027)

[FMJ01] F. Fauquant-Millet and A. Joseph, Sur les semi-invariants d'une sous-algèbre parabolique d'une algèbre enveloppante quantifiée, Transformation Groups 6 (2001), no. 2, 125-142. MR1835668 (2002f:17020)

[Hel78] S. Helgason, Differential geometry, Lie groups, and symmetric spaces, Pure and Applied Mathematics, no. 80, Academic Press, New York, 1978. MR514561 (80k:53081)

[IK05] N.Z. Iorgov and A.U. Klimyk, Classification theorem on irreducible representations of the q-deformed algebra $U_{q}^{\prime}\left(s o_{n}\right)$, Int. J. Math. Math. Sci. 2005 (2005), no. 2, 225-262. MR2143754 (2006c:17021)

[JL94] A. Joseph and G. Letzter, Separation of variables for quantized enveloping algebras, Amer. J. Math. 116 (1994), 127-177. MR1262429 (95e:17017)

[Jos95] A. Joseph, Quantum groups and their primitive ideals, Ergebnisse der Mathematik und ihrer Grenzgebiete, Springer-Verlag, Berlin, 1995. MR1315966 (96d:17015)

[Kéb99] M.S. Kébé, Sur la classification des OO-algèbres quantiques, J. Algebra 212 (1999), 626659. MR 1676857 (2000g:17018) 
[Kol] S. Kolb, Quantum symmetric pairs and the reflection equation, Algebr. Represent. Theory, in press, DOI 10.1007/s10468-008-9093-6.

[Let99a] G. Letzter, Harish-Chandra modules for quantum symmetric pairs, Representation Theory, An Electronic Journal of the AMS 4 (1999), 64-96. MR.1742961 (2001h:17025)

[Let99b] _ Symmetric pairs for quantized enveloping algebras, J. Algebra 220 (1999), 729767. MR 1717368 (2001b:17026)

[Let02] Coideal subalgebras and quantum symmetric pairs, New directions in Hopf algebras (Cambridge), MSRI publications, vol. 43, Cambridge Univ. Press, 2002, pp. $117-$ 166. MR 1913438 (2003g:17025)

[Let03] Quantum symmetric pairs and their zonal spherical functions, Transformation Groups 8 (2003), 261-292. MR1996417 (2004h:17017)

[Let04] , Quantum zonal spherical functions and Macdonald polynomials, Adv. Math. 189 (2004), 88-147. MR2093481 (2005i:33019)

[Let08] - Invariant differential operators for quantum symmetric spaces, Mem. Amer. Math. Soc. 193 (2008), no. 903. MR2400554

[Mac00] I.G. Macdonald, Orthogonal polynomials associated with root systems, Séminaire Lotharingien de Combinatoire 45 (2000), 45 pp. MR.1817334 (2002a:33021)

[Nou96] M. Noumi, Macdonald's symmetric polynomials as zonal spherical functions on some quantum homogeneous spaces, Adv. Math. 123 (1996), 16-77. MR1413836 (98a:33004)

[NS95] M. Noumi and T. Sugitani, Quantum symmetric spaces and related q-orthogonal polynomials, Group theoretical methods in physics (Singapore) (A. Arima et al., ed.), World Scientific, 1995, pp. 28-40. MR 1413733 (97h:33033)

[OS05] A.A. Oblomkov and J.V. Stokman, Vector valued spherical functions and MacdonaldKoornwinder polynomials, Compos. Math. 141 (2005), no. 5, 1310-1350. MR2157139 (2006f:33022)

[OV94] A.L. Onishchik and E.B. Vinberg, Lie groups and Lie algebras III, Encyclopeadia of Mathematical Sciences, no. 41, Springer-Verlag, Berlin, 1994. MR1349140 (96d:22001)

[Ros90] M. Rosso, Analogues de la forme de Killing et du théorème d'Harish-Chandra pour les groupes quantiques, Ann. scient. Éc. Norm. Sup. 23 (1990), 445-467. MR 1055444 (93e:17026)

Department of Mathematics, Virginia Tech, Blacksburg, Virginia 24061

Current address: School of Mathematics and Maxwell Institute for Mathematical Sciences, The University of Edinburgh, JCMB, The King's Buildings, Mayfield Road, Edinburgh, EH9 3JZ, United Kingdom

E-mail address: stefan.kolb@ed.ac.uk

Department of Mathematics, Virginia Tech, Blacksburg, Virginia 24061

E-mail address: gletzter@verizon.net 